\title{
Psychische Belastung und deren Herausforderungen
}

\author{
Yvonne Ferreira' ${ }^{1}$ Joachim Vogt ${ }^{2}$
}

Angenommen: 5. November 2021 / Online publiziert: 7. Dezember 2021

(C) Der/die Autor(en) 2021

\section{Zusammenfassung}

Psychische Belastung stellt sowohl für die Praxis als auch für die Wissenschaft eine aktuelle Herausforderung dar. Einerseits zeigen Veröffentlichungen, dass der Sprachgebrauch und das Verständnis der Einwirkungen und Auswirkungen des Belastungs-Beanspruchungs-Konzeptes - trotz DIN EN ISO 10075-1 - uneinheitlich sind und dies in der Praxis zu Verunsicherung, in der Wissenschaft zu Missverständnissen führt. Andererseits mangelt es an konkreten Untersuchungen zu dringend notwendigen Grenzwerten psychischer Belastung. Der vorliegende Artikel stellt die Entwicklung eines Prozessmodells zur Untersuchung von Grenzwerten psychischer Belastung (kurz Arbeitsmodell) vor, in welchem die relevanten Elemente definiert sind und diskutiert werden. $\mathrm{Zu}$ einer möglichst einheitlichen Erfassung von Belastungsfaktoren und Ressourcen werden mittels qualitativer Forschungsmethoden Kategoriensysteme entwickelt und vorgestellt, um die Daten statistischen Vorhersagemodellen zugänglich zu machen.

Praktische Relevanz Die Betriebe sind gemäß Gesetzgebung angewiesen, regelmäßige Risikobeurteilungen der vorhandenen psychischen Belastung der Arbeitsplätze vorzunehmen. Um ein Risiko beurteilen zu können, fehlt jedoch die wissenschaftliche Basis in Form von Grenzwerten. Diese den Betrieben an die Hand zu geben ist ein dringendes und notwendiges Vorhaben.

Schlüsselwörter Grenzwerte $\cdot$ Psychische Belastung $\cdot$ Psychische Fehlbelastung $\cdot$ Gefährdungsbeurteilung $\cdot$ Arbeitsschutz

\section{Mental load and its challenges}

\begin{abstract}
Mental load is a current challenge for both practice and science. On the one hand, publications show that the use of language and the understanding of the effects and impacts of the stress-strain concept are inconsistent—despite DIN EN ISO 10075-1 - and this leads to uncertainty in practice and misunderstandings in science. On the other hand, there is a lack of concrete studies on urgently needed limit values for mental load. This article presents the development of a process model for the investigation of threshold values of mental load, in which the relevant elements are defined and discussed. In order to record loading factors and resources as uniformly as possible, categorical systems are developed and presented using qualitative research methods in order to make the data accessible to statistical prediction models.

Practical Relevance Legislation requires companies to carry out regular risk assessments of the existing mental load in the workplace. However, there is no scientific basis in the form of limit values for assessing the risk. It is an urgent and necessary project to provide these to the companies.
\end{abstract}

Keywords Limit values $\cdot$ Mental stress $\cdot$ Mental aversive load $\cdot$ Risk assessment $\cdot$ Occupational safety

Prof. PD Dr. habil. Yvonne Ferreira

yvonne.ferreira@fom.de

1 Institut für Wirtschaftspsychologie IWP, FOM -

Hochschule für Oekonomie und Management,

Franklinstraße 52, 60486 Frankfurt am Main, Deutschland

2 Forschungsgruppe Arbeits- und Ingenieurpsychologie, Technische Universität Darmstadt, Darmstadt, Deutschland 


\section{Einführung}

Im Zuge der fortschreitenden Globalisierung und Digitalisierung verändern sich die Arbeitsbedingungen und Arbeitsprozesse für Beschäftigte. In einigen Wirtschaftsbereichen werden körperliche durch geistige Arbeitsanforderungen weitestgehend ersetzt, wodurch allgemein zwar eine Reduzierung körperlicher, dafür jedoch ein Anstieg an psychischer Belastung zu verzeichnen ist (Junghanns et al. 2013; Wulf et al. 2017; Cernavin et al. 2018; Schuller 2020).

Aus arbeitswissenschaftlicher und -psychologischer Sicht ist es von großer Relevanz, physische und psychische Gesundheit durch die Vermeidung von Beeinträchtigungen nicht nur zu erhalten, sondern auch zu verbessern. Dieses Interesse sollte nicht allein bei den Beschäftigten und der Gesellschaft insgesamt liegen, sondern auch bei den Unternehmen, da die Gesundheit der Beschäftigten beispielsweise direkt mit Fehlhandlungen eng zusammenhängt aber auch mit deren Leistungsfähigkeit, die wiederum einen großen Einfluss auf die Qualität der Arbeitsergebnisse und den Erfolg von Unternehmen hat (Vogt et al. 2002, 2010; Möller et al. 2008; Pennig und Vogt 2008; Böcker et al. 2009; Wulf et al. 2017).

Psychische und physische Gesundheit werden vom Arbeitsschutzgesetz (Gesetz über die Durchführung von Maßnahmen des Arbeitsschutzes zur Verbesserung der Sicherheit und des Gesundheitsschutzes der Beschäftigten bei der Arbeit, ArbSchG 2015) auf die gleiche Ebene gestellt. Den Unternehmen wird auferlegt, Gefährdungen für das Leben sowie die physische und die psychische Gesundheit der Beschäftigten zu vermeiden und verbleibende Gefährdungen so gering wie möglich zu halten ( 4 , ArbSchG 2015).

Konkret werden Unternehmen durch $\S 5$ ArbSchG verpflichtet, eine Gefährdungsbeurteilung durchzuführen und notwendige Maßnahmen des Arbeitsschutzes einzuleiten ( 55 Abs. 1). Hierbei verweist der Gesetzgeber auf die Gefährdung durch die psychische Belastung bei der Arbeit ( $\$ 5$ Abs. 3 Nr. 6), auf die im Folgenden eingegangen wird. Dass hier nach wie vor Handlungsbedarf besteht und die Umsetzung von Gefährdungsbeurteilungen in vielen Betrieben noch nicht ausreichend ist, zeigen aktuelle Standortbestimmungen (beispielsweise Beck et al. 2012; Schuller 2020). Für spezielle Branchen, beispielsweise Banken, wird jedoch angegeben, dass neun von zehn Beschäftigte in Unternehmen tätig sind, die Gefährdungsbeurteilungen durchführen. Jedoch umfassen nicht alle Gefährdungsbeurteilungen die Beurteilung der psychischen Belastung (Cernavin et al. 2018). Die Beurteilung der psychischen Belastung ist für Betriebe deutlich erschwert, da es bisher keine Orientierungshilfen wie Grenzwerte gibt. Zwar werden in zahlreichen Publikationen „Belastungsgrenzen“ vorausgesetzt (Bauer et al. 2021; Haas und Reblin 2021), aber nicht näher konkretisiert.

\subsection{Begriffsbestimmungen und Konzeption}

Das Konstrukt der psychischen Belastung wird in der Literatur weder einheitlich bezeichnet noch verstehen Wissenschaftler/-innen, Institutionen, Unternehmen, Gesetzgebung und/oder Beschäftigte das Gleiche darunter. Dies ist verwunderlich, da die Terminologie sowie eine Definition bereits in ISO 10075 (1991) und aktuell in der DIN EN ISO 10075-1 (2018) auf nationaler, europäischer und internationaler Ebene festgeschrieben ist. Die Norm fordert, von psychischer Belastung im Singular zu sprechen, denn die Definition besagt, es handele sich dabei um die Gesamtheit aller erfassbarer Einwirkungen die von außen auf den Menschen einwirken und nicht um einzelne Komponenten oder Facetten dieser Belastung. Dieses Verständnis ist nicht neu, denn beispielsweise Nachreiner diskutiert bereits 2008 diese Frage (Nachreiner 2008). In der vorliegenden Publikation wird psychische Belastung daher konsequent im Singular referiert, unabhängig von abweichenden (zitierten) Publikationen oder der Verwendung durch das für den Arbeitsschutz und die Gefährdungsbeurteilung zuständige BMAS und seine nachgeordneten Institutionen (z. B. BAuA 2017 und GDA 2018).

Die Uneinigkeit in Hinblick auf die zu verwendende Terminologie psychischer Belastung sowie deren Definitionen und Abgrenzungen führt zu Verständnisschwierigkeiten und fehlenden Vergleichsmöglichkeiten nicht nur zwischen verschiedenen Fachdisziplinen, sondern auch innerhalb dieser Fachdisziplinen, einschließlich der Arbeitspsychologie. Daher ist es unter anderem ein Anliegen dieser Forschungsarbeit, ein generalisierbares konzeptuelles Prozessmodell zur Untersuchung von Grenzwerten psychischer Belastung (kurz Arbeitsmodell) für diese und weitere Arbeiten zu diesem Konstrukt zu entwickeln, welches die Zusammenhänge und Abgrenzungen der Konstrukte untereinander aufzeigt und diese eindeutig definiert und modellhaft illustriert.

Die im Folgenden dargestellten Definitionen sowie das darauf basierende Arbeitsmodell berücksichtigen das klassische Belastungs-Beanspruchungs-Konzept (Rohmert und Rutenfranz 1975) sowie die Normen DIN EN ISO 10075-1 $(2018)^{1}$ (Ergonomische Grundlagen bezüglich psychischer Arbeitsbelastung) und DIN EN ISO 6385 (2016) ${ }^{2}$ (Grundsätze der Ergonomie für die Gestaltung von Arbeitssystemen), die etablierten wissenschaftlichen Kenntnisse sowie die Stellungnahmen der GDA und die Forschungen der BAUA (obwohl diese Quellen begrifflich und konzeptuell teilweise inkompatibel sind).

In den folgenden Unterkapiteln werden die Elemente, die sich im Arbeitsmodell (Abschn. 1.1.8) zusammenfügen,

\footnotetext{
1 Wie auch die Vorläuferversionen.

2 Wie auch die Vorläuferversionen.
} 


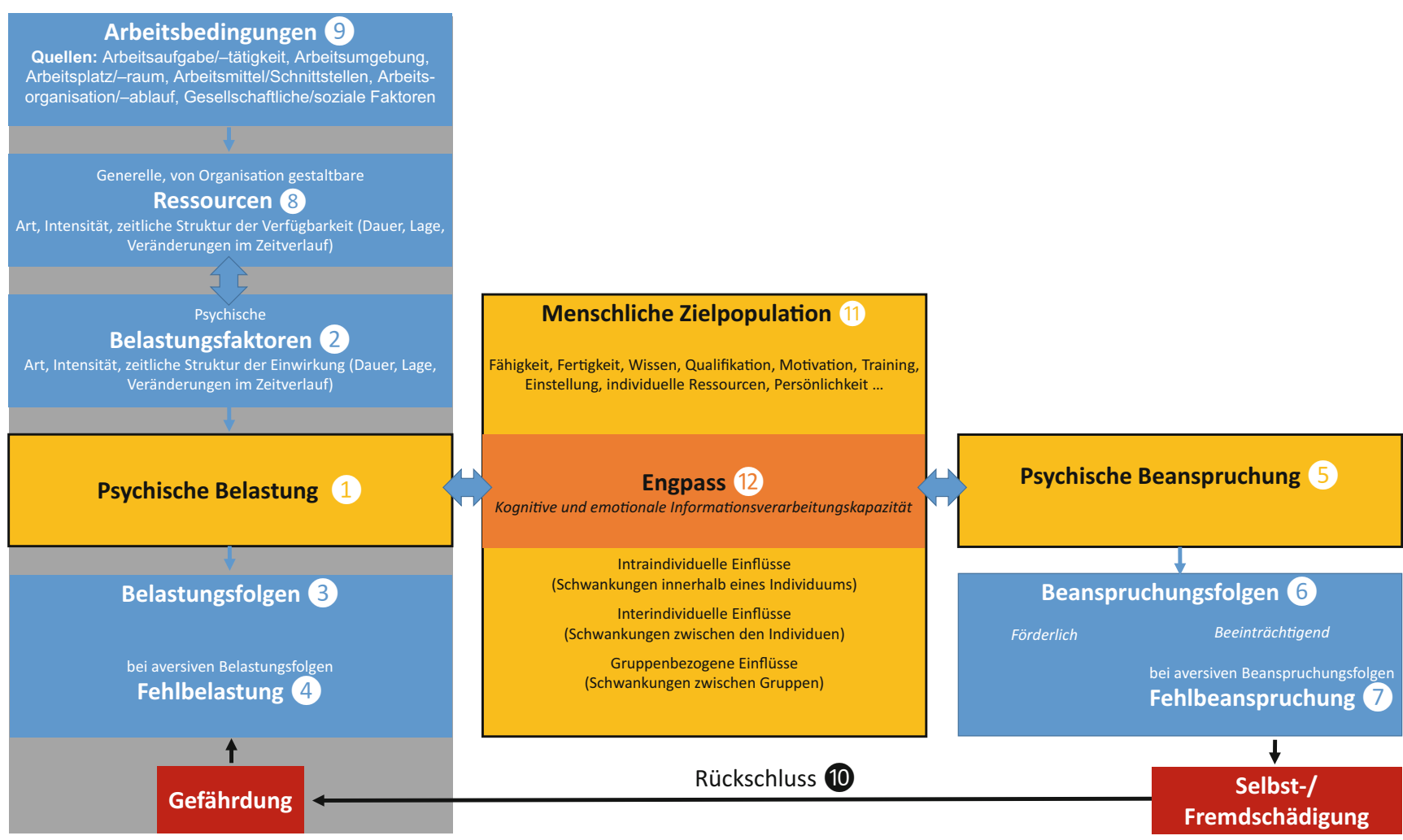

Abb. 1 Prozessmodell (kurz Arbeitsmodell) zur Untersuchung von Grenzwerten psychischer Belastung

Fig. 1 Process model for the investigation of limit values of mental stress

vorab einzeln definiert und erklärt. Die Zahlen innerhalb der Kreise verweisen auf das Arbeitsmodell in Abb. 1.

\subsubsection{Psychische Belastung}

Die Begriffsdefinition der psychischen Belastung (1) findet sich aktuell in der DIN EN ISO 10075-1 (2018). Hintergrund der Definition ist das Belastungs-BeanspruchungsKonzept der Ergonomie von Rohmert und Rutenfranz (1975), welches den Zusammenhang zwischen der (allgemeinen) Belastung am Arbeitsplatz als Einwirkungsgröße und die daraus resultierenden Auswirkungen im Menschen beschreibt und auf einem klassischen Stimulus-OrganismResponse (S-O-R)-Modell beruht.

Gemäß DIN EN ISO 10075-1 ist die psychische Belastung ,die Gesamtheit aller erfassbaren Einflüsse, die von außen auf den Menschen zukommen und psychisch auf ihn einwirken" (DIN EN ISO 10075-1 2018, S. 6).

DIN EN ISO 10075-1 verwendet den Begriff psychische Belastung ausschließlich im Singular, da die psychische Belastung die Gesamtheit ${ }^{3}$ aus verschiedenen Belastungsfaktoren (2) im jeweiligen Wirkungsprozess darstellt. Belastungsfaktoren sind hierbei sämtliche Einwirkfaktoren.

\footnotetext{
${ }^{3}$ Und davon kann es konzeptuell nur eine geben.
}

Damit erweitert die DIN EN ISO 10075-1 das von Luczak und Rohmert (1997) geprägte Verständnis der Belastungsfaktoren. Luczak und Rohmert (1997) unterscheiden die Begriffe Belastungsgrößen und Belastungsfaktoren hinsichtlich der vorliegenden Skalenniveaus. Belastungsgrößen können auf metrischem Niveau, Belastungsfaktoren lediglich auf ordinalem oder nominalem Niveau erhoben werden. DIN EN ISO 10075-1 ändert diese Auslegung zugunsten eines allgemeineren, im Normungsprozess konsensuell erarbeiteten Verständnisses.

In der Tat fügt sich diese Begrifflichkeit sehr gut in das Belastungs-Beanspruchungs-Konzept ein. Die Definition von Faktor im Duden (www.duden.de) lautet ,etwas, was in einem bestimmten Zusammenhang bestimmte Auswirkungen hat". Die psychische Belastung ist als Konstrukt genau diese Gesamtheit, wobei das Wort (Belastungs-)Faktor darauf hinweist, dass die einzelnen Komponenten der psychischen Belastung ,in einem bestimmten Zusammenhang" (also je nach Zusammensetzung, Intensität und zeitlicher Struktur) „,bestimmte Auswirkungen“ haben.

\subsubsection{Belastungsfolgen und Psychische Fehlbelastung}

Sowohl die DIN EN ISO 10075-1 als auch die DIN EN ISO 6385 definieren (psychische) Fehlbelastung nicht, da Belastung als neutral festgelegt ist und die Gesamtheit aller 
Einwirkfaktoren umfasst. Das hier vorgestellte Arbeitsmodell jedoch legt auf psychische Fehlbelastung einen Schwerpunkt, der sich einerseits aufgrund des Untersuchungsgegenstands erklärt (Grenzwerte psychischer Belastung) und andererseits aufgrund der Forderungen des Arbeitsschutzgesetzes. Der Gesetzgeber verpflichtet Unternehmen mit § 5 zur Beurteilung der Arbeitsbedingungen, um vorhandene Gefährdungen einzuschätzen ( $\$ 5(1))$. Fokussiert werden die Arbeitsbedingungen - und damit unbestritten die Belastung. Ebenfalls in $\S 5$ (3) 6 wird explizit die psychische Belastung bei der Arbeit genannt. Noch konkreter heißt es in $\$ 5$ (2): „Bei gleichartigen Arbeitsbedingungen ist die Beurteilung eines Arbeitsplatzes oder einer Tätigkeit ausreichend". Es geht also nicht um individuelle Beanspruchungsfolgen bei dieser Forderung, sondern um Belastung und deren potenziell aversive Folgen (Belastungsfolgen), basierend auf den bisherigen Erfahrungen. Es geht um die Frage, ob psychische Belastung aversive Konsequenzen hat, welche sich als Fehlbelastung und damit als Gefährdung bezeichnet darstellen. Mit diesem Gedankengang erfüllt das Arbeitsmodell die Forderungen des Arbeitsschutzgesetzes. Dass die Gefährdung jedoch nur über Beanspruchungsfolgen und potenziell aversive Folgen definiert werden kann, ist selbstredend. Dennoch ist es aus zwei Gründen wichtig, Fehlbelastung und Gefährdung auf der Belastungsseite abzubilden:

1. Organisationen können psychische Belastung gestalten. Sie sollen derart ausfallen, dass sie zu keinen aversiven Folgen, bestenfalls zu keinen Gefährdungen der Beschäftigten führt. Es geht hier demnach um kollektiven Arbeitsschutz - nicht um individuellen, wie dieser auf der Beanspruchungsseite des Modells anzusiedeln wäre. Daher unterscheidet das unter Abschn. 1.1.8 dargestellte Arbeitsmodell eine allgemeinpsychologische (linker Teil, grau unterlegt) und eine differentialpsychologische (rechter Teil) Betrachtungsweise. Dies ist für spätere empirische Erhebungen, Interpretationen des Datenmaterials und der Verwendung im Arbeitsschutz von großer Bedeutung.

2. Das letztendliche, hier aber noch nicht erreichbare Ziel des gesamten Forschungsvorhabens ist es, mögliche Strategien der Ermittlung von Grenzwerten oder Gefährdungen aufzuzeigen - und diese auf der Belastungsseite $\mathrm{zu}$ verorten.

Die Argumentation, Belastungsfolgen seien gleichzusetzen mit Beanspruchungsfolgen und damit im Arbeitsmodell doppelt abgebildet, ist nicht korrekt. Korrekt ist, dass Belastungsfolgen nur über Beanspruchungsfolgen zu definieren sind. Die Unterscheidung zwischen Belastungsund Beanspruchungsfolgen ist auf die Abgrenzung zwischen allgemeinpsychologischer und differentialpsychologischer Betrachtung im Arbeitsmodell zurückzuführen. Be- anspruchungsfolgen sind - gemäß differentialpsychologischer Sicht - individuell. Das Arbeitsmodell jedoch postuliert, dass aversive Belastungsfolgen für nahezu alle Personen einer vorab definierten menschlichen Zielpopulation zur Fehlbelastung und damit zur Gefährdung werden. Die im Modell dargestellte Fehlbelastung ist bestenfalls bereits aus vorausgehenden Untersuchungen der Beanspruchungsfolgen bestimmter Belastungskomponenten validiert - es handelt sich im Arbeitsmodell dementsprechend um einen Rückkopplungsprozess, genauer betrachtet um ein Prozessmodell zur Untersuchung von Grenzwerten psychischer Belastung.

DIN EN ISO 10075-1 räumt ein, dass psychische Belastung positiv oder negativ einwirken kann, und somit zu einem Anstieg oder zu einer Verringerung psychischer Beanspruchung führt (siehe Abschn. 1.1.3). Dies bedeutet, dass psychischer Belastung eine Beanspruchung folgt, weshalb im Arbeitsmodell vereinfachend von Belastungsfolgen (3) gesprochen wird, wissentlich, dass es sich genau genommen um Beanspruchungsfolgen handelt (weil die direkte Folge einer Belastung die Beanspruchung ist). Gemäß DIN EN ISO 10075-1 wird daher von Beanspruchungsfolgen gesprochen (als Auswirkung der Beanspruchung, die selbst wiederum eine Folge der Belastung darstellt). Da für das Arbeitsmodell - und damit für die künftige empirische Datenerhebung zur Untersuchung von Grenzwerten psychischer Belastung - genau dieser Zwischenschritt (wann hat psychische Belastung eine Gefährdung zur Folge) relevant ist, wird statt der korrekten, aber umständlichen Bezeichnung „Belastungs-Beanspruchungsfolgen“, vereinfachend von (indirekten) Belastungsfolgen gesprochen. Analog zu den in DIN EN ISO 10075-1 definierten beeinträchtigenden bzw. förderlichen Beanspruchungsfolgen, unterteilen sich die Belastungsfolgen in positiv wirkend (im Sinne einer optimierenden Einwirkung auf die psychische Beanspruchung und ihre Folgen) und Fehlbelastung (im Sinne eines negativen Einwirkens auf die psychische Beanspruchung).

In einem Bericht des Länderausschusses für Arbeitsschutz und Sicherheitstechnik (LASI 2002, S. 11) wird psychische Fehlbelastung (4) definiert als ,[...] BelastungEN [...], die in ihrer Ausprägung mit einer hohen Wahrscheinlichkeit bei Beschäftigten zu [...] Beeinträchtigungen führen“. Diese Beschreibung ist sehr allgemein gehalten, weist jedoch auf negative Einwirkung von bestimmten Belastungskomponenten (und daher dort als Fehlbelastung bezeichnet) hin. Für eine konkrete Definition bietet die DIN EN ISO 10075-1 weitere Anhaltspunkte: „Eine psychische Belastung, die das menschliche Vermögen zur Informationsverarbeitung übersteigt, wird direkt zu einer fehlerhaften menschlichen Leistung führen“ (S. 10). Weiterhin ist von „dysfunktionalen Anforderungen“ die Rede. 
Basierend auf dem Belastungs-Beanspruchungs-Konzept, DIN EN ISO 10075-1 sowie der GDA wird psychische Fehlbelastung im Arbeitsmodell folgendermaßen definiert:

Psychische Fehlbelastung ist eine Konstellation oder Gesamtheit von Belastungsfaktoren, die von außen auf den Menschen einwirken und aufgrund ihrer Art, Intensität, zeitlichen Struktur der Einwirkung (Dauer, Lage, Veränderungen im Zeitverlauf) und/oder Kombination die menschliche kognitive und emotionale Verarbeitungskapazität der prospektiven Zielpopulation übersteigen. Psychische Fehlbelastung führt damit bei einer hinreichend geeigneten Zielpopulation mit hoher Wahrscheinlichkeit zu aversiven Konsequenzen für die Betroffenen selbst und/oder ihre Umwelt.

\subsubsection{Psychische Beanspruchung}

Die Auswirkungen psychischer Belastung im Menschen werden psychische Beanspruchung (5) genannt und sind unter anderem abhängig von der Art und Intensität sowie der zeitlichen Dauer, Lage und Veränderungen der Belastung (DIN EN ISO 10075-1). Psychische Beanspruchung ist ,die unmittelbare (nicht die langfristige) Auswirkung der psychischen Belastung im Individuum in Abhängigkeit von seinen jeweiligen überdauernden und augenblicklichen Voraussetzungen, einschließlich der individuellen Bewältigungsstrategien.“ (DIN EN ISO 100075-1 2018, S. 3).

Da es sich bei den Forderungen des Arbeitsschutzgesetzes eindeutig um Belastungsfaktoren sowie deren konkrete Gestaltung und Gestaltbarkeit handelt und nicht nach Auswirkungen auf den Organismus, sondern nach Gefährdung für die prospektive Zielpopulation gefragt wird, wird in dieser Forschungsarbeit die psychische Beanspruchung nicht fokussiert betrachtet (siehe 1.1.8 Arbeitsmodell). Selbstredend ist psychische Beanspruchung jedoch bei den Betrachtungen zwingend notwendig, um Rückschlüsse auf psychische Belastung zu ermöglichen, wie weiter oben bei der Charakterisierung der psychischen Fehlbelastung dargestellt. Anders, als im Rückschluss von der Beanspruchung auf die Belastung, kann psychische Fehlbelastung nicht erfasst werden.

\subsubsection{Beanspruchungsfolgen und Psychische Fehlbeanspruchung}

Psychische Beanspruchung ist ebenfalls definitionsgemäß neutral, kann jedoch zu förderlichen bzw. beeinträchtigenden Beanspruchungsfolgen führen (6). Definitionen zu psychischer Fehlbeanspruchung (7) $\mathrm{zu}$ finden, ist nahezu unmöglich. Häufig wird Fehlbeanspruchung lediglich durch Rückschluss auf Beanspruchungsfolgen beschrieben und definiert, wie beispielsweise bei Joiko et al. (2010).

Die GDA definiert, dass psychische Fehlbeanspruchung dann entsteht, ,wenn die Leistungsvoraussetzungen von Beschäftigten bei ihrer Arbeit quantitativ und/oder qualitativ über- oder unterfordert werden“ (GDA 2018, S. 28). Dies ist jedoch nicht korrekt formuliert, denn es entsteht eine Belastung, die in Fehlbeanspruchung resultiert.

Die DIN selbst spricht von Auswirkungen, die beeinträchtigend oder förderlich sein können. Diese Auswirkungen werden nochmals unterteilt hinsichtlich ihrer Dauer (wenige Minuten bis komplettes Berufsleben), dem Einsetzen der Auswirkungen (sofort bis verzögert) sowie der Dauer der Auswirkung (vorübergehend bis chronisch). Im vorliegenden Arbeitsmodell ist Fehlbeanspruchung definiert als direkte Auswirkung einer Fehlbelastung im Organismus, die zu aversiven Folgen führt.

\subsubsection{Ressourcen}

Zahlreiche Modelle postulieren nachvollziehbar einen $\mathrm{Zu}$ sammenhang zwischen verfügbaren organisationalen Ressourcen (8) und Belastung (beispielhaft Wickens 2008; Bouveresse et al. 2011; Schmidt und Diestel 2013; Krehl und Balfe 2014; Uhle und Treier 2015; Wegge et al. 2018). Ebenso erwähnt DIN EN ISO 10075-1 Ressourcen im Zusammenhang mit psychischer Belastung. Die Evidenz der Wirksamkeit von Ressourcen, und sei es lediglich als moderierende Variable, macht es notwendig, diese im Rahmen eines kollektiven (nicht individualisierten) Arbeitsschutzes in die Betrachtung von Grenzwerten psychischer Belastung einzubeziehen (vgl. Abschn. 1.1). Hierbei muss es sich auf der Belastungsseite um die Betrachtung genereller Ressourcen handeln, also solche, die einerseits von der Organisation gestaltbar sind und andererseits Belastungsfaktoren zuzuordnen sind (individuelle Ressourcen finden sich im Bereich der Beschäftigten im Modell wieder). In Anbetracht der Definition von psychischer Fehlbelastung (Abschn. 1.1.2) kommen Überlegungen in Frage, bei denen generelle, von der Organisation gestaltbare Ressourcen die Beanspruchungsfolgen bei einem Großteil der Zielpopulation intervenieren können.

Ressourcen - so die Annahme im Arbeitsmodell - wirken im Zusammenspiel mit den Arbeitsbedingungen und den Belastungsfaktoren vermittelnd und kompensatorisch und gehören somit in die Betrachtung als eine Komponente der psychischen Belastung. Man kann sogar so weit gehen zu sagen, dass sich auswirkende Ressourcen zu psychischer Belastung gehören. Hierzu müssen Ressourcen unterteilt werden von ausreichend vorhanden über begrenzt vorhanden bis nicht vorhanden unter Berücksichtigung der Art, Intensität und der zeitlichen Struktur der Verfügbarkeit. Erst, wenn es in der menschlichen Zielpopulation zu einem Eng- 
pass kommt, kann der Rückschluss gezogen werden, dass die zur Verfügung stehenden organisationalen Ressourcen nicht ausreichend sind.

Zur Prüfung solcher Zusammenhänge ist zunächst eine Definition des Ressourcenbegriffs erforderlich. Herausfordernd sind hierbei die interdisziplinär stark unterschiedlichen Auslegungen. Schubert und Knecht (2015, S. 3) definieren: „Ressourcen sind positive personale, soziale und materielle Gegebenheiten, Objekte, Mittel, Merkmale bzw. Eigenschaften, die Personen nutzen können, um alltägliche oder spezifische Lebensanforderungen wie auch psychosoziale Entwicklungsaufgaben zu bewältigen, um psychische wie physische Bedürfnisse und eigene Wünsche zu erfüllen, Lebensziele zu verfolgen und letztlich Gesundheit und Wohlbefinden zu erhalten bzw. wieder herzustellen“. Grundsätzlich wird zwischen personalen Ressourcen, Umweltressourcen und arbeitsbezogenen Ressourcen unterschieden. Das Arbeitsmodell benötigt eine praktikablere Definition von Ressourcen, die sich vor allem darauf bezieht, dass die Ressourcen vom Unternehmen für nahezu alle Beschäftigte (also kollektiv) gestaltbar sein müssen:

Generelle, von der Organisation gestaltbare Ressourcen sind personale, soziale und materielle Faktoren, die aufgrund Art, Intensität, zeitliche Struktur der Verfügbarkeit (Dauer, Lage, Veränderungen im Zeitverlauf) geeignet sind, Belastungsfolgen zu optimieren.

\subsubsection{Arbeitsbedingungen}

Eines der Ziele dieser Forschungsarbeit besteht darin, Belastungsfaktoren und Ressourcen bezogen auf die Arbeitsbedingungen (9) in Kategoriensysteme (siehe weiter unten) zu unterteilen, um zur Identifikation von Fehlbelastungskonstellationen statistische Modelle entwickeln zu können. $\mathrm{Zu}$ diesem Zweck werden Interviews in verschiedenen Berufsgruppen durchgeführt (siehe Abschn. 2). Ein Ergebnis der Untersuchung - welches hier vorweg referiert werden muss, um das entwickelte Arbeitsmodell zu verdeutlichen zeigt, dass die Quellen von Belastungsfaktoren ebenfalls auch die potenziellen Quellen von (durch das Unternehmen gestaltbaren) Ressourcen sind (siehe Abschn. 3). Als Beispiel sei die Arbeitsumgebung genannt und hier die Temperatur als neutraler Belastungsfaktor. Eine Ressource aus der Arbeitsumgebung könnte beispielsweise eine Klimaanlage oder eine Heizung sein. Sowohl der Belastungsfaktor als auch die Ressource stammen in diesem Beispiel aus der Arbeitsumgebung. Die Ressourcen können selbstverständlich auch aus anderen Quellen stammen. Beispielsweise könnte die Autonomie der Kontrolle der vorhandenen Belastung (hier die Möglichkeit, Temperatur selbst zu wählen), als Ressource betrachtet werden. Dann läge die Quelle in Arbeitsorganisation und -ablauf.
Sowohl DIN EN ISO 10075-1 als auch DIN EN ISO 6385 nennen Quellen von Belastungsfaktoren. Zudem sind diese in der arbeitswissenschaftlichen und -psychologischen Literatur aufgeführt (beispielsweise Joiko et al. 2010, S. 21; Nerdinger et al. 2019, S. 412) und finden sich auch in der GDA-Leitlinie (2018) zur ,Beratung und Überwachung bei psychischer Belastung am Arbeitsplatz“. Zusammengefasst ergeben diese Informationen die folgenden Quellen:

- Arbeitsaufgabe und -tätigkeit

- Arbeitsumgebung

- Arbeitsplatz und -raum

- Arbeitsmittel und Schnittstellen

- Arbeitsorganisation und -ablauf

- Gesellschaftliche/soziale Faktoren.

\subsubsection{Beschäftigte (Prospektive Zielpopulation)}

Der Fokus dieser Forschungsarbeit liegt auf psychischer Belastung und Fehlbelastung und damit auf einer potenziellen Gefährdung durch die Arbeit und ihre Bedingungen, die es mit Mitteln der Arbeitsgestaltung zu vermeiden gilt. Eine Gefährdung kann für eine prospektive Zielpopulation vermieden werden, aber nur dann, wenn von aversiven Beanspruchungsfolgen auf Gefährdungen zurückgeschlossen werden kann (10). Unter der hier unterstellten S-O-R-Konzeption (vgl. Abschn. 1.1.1) ist es unvermeidlich, auch den Faktor Mensch mit in die Betrachtungen einzuschließen, denn lediglich über den Menschen können gesundheitliche Beanspruchungsfolgen definiert werden. Das Arbeitsmodell sieht eine menschliche Zielpopulation vor, die eine Gruppe von Menschen umfasst, die theoretisch von ihrer Qualifikation her in der Lage ist, die Arbeitsaufgabe zu erfüllen. Hierzu sind beispielsweise bestimmte Qualifikationen, Fähigkeiten und Fertigkeiten zwingende Voraussetzungen. Beispielsweise müsste eine Zielpopulation für die Tätigkeit „Pilot/-in“ andere Voraussetzungen mit sich bringen als eine Zielpopulation für die Tätigkeit „Landwirt/-in“.

Die menschliche Zielpopulation kann hinsichtlich intraindividueller, interindividueller und gruppenbezogener Variationen betrachtet und analysiert werden. Intraindividuelle Einflüsse beschreiben die Schwankungen innerhalb eines Individuums, beispielsweise in der Aufmerksamkeitsleistung über eine Arbeitsschicht. Interindividuelle Einflüsse beschreiben die Schwankungen zwischen den Individuen, wie beispielsweise der Intelligenzquotient. Die gruppenbezogenen Einflüsse beinhalten die Schwankungen zwischen Gruppen, die beispielsweise Normen und Werte vorgeben und die Motivation der Gruppenmitglieder aber auch der Fremdgruppen beeinflussen können.

In der Arbeitswissenschaft hat sich basierend auf dem Belastungs-Beanspruchungs-Konzept eine engpassbezoge- 
ne Betrachtungsweise der menschlichen Funktions- und Reaktionsbereiche (Perzeption, Kognition, Motorik, Emotionalität) entwickelt (Rohmert 1984; Oesterreich 2001). Darunter wird verstanden, dass der menschliche Organismus bei der Arbeit Beschränkungen hinsichtlich der Wahrnehmung, der Informationsverarbeitung, der Emotionsregulation und der körperlichen Bewegungsmöglichkeiten unterliegt. Engpässe (12) sind entscheidend zur Bewertung einer Belastung und entscheidend für die Definition der Fehlbelastung. DIN EN ISO 10075-1 bezieht die psychische Belastung auf kognitive und emotionale Vorgänge im Menschen, weshalb im vorliegenden Arbeitsmodell in diesen Bereichen die Engpässe fokussiert werden. Ob sich die Fokussierung auf Informationsverarbeitungskapazitäten bewähren wird, muss sich erst durch intensivere Forschung mit dem Arbeitsmodell ergeben, denn Engpässe psychischer Belastung können ebenso in anderen Bereichen - beispielsweise muskulär - auftreten. Zum jetzigen Zeitpunkt ist die enge Anlehnung an DIN EN ISO 10075-1 ausschlaggebend. Der Engpass entsteht, wenn die Belastung die vorhandenen Möglichkeiten zur Aufgabenerfüllung übersteigt und damit zur Fehlbelastung wird.

\subsubsection{Arbeitsmodell}

Das aus den vorherigen Überlegungen und Definitionen abgeleitete Prozessmodell zur Untersuchung von Grenzwerten psychischer Belastung (hier kurz Arbeitsmodell) ist die Grundlage der vorliegenden Forschungsarbeit (siehe Abb. 1).

Die Basis dieses Arbeitsmodells ist das klassische Belastungs-Beanspruchungs-Konzept (schwarz umrandete Bereiche des Arbeitsmodells), welches sich bei den weiterführenden Betrachtungen jedoch ausschließlich auf psychische Belastung und Beanspruchung bezieht (die selbstverständlich auch aus körperlicher Belastung resultieren können).

Arbeitsaufgabe und -tätigkeit, -umgebung, -platz und -raum, -mittel und Schnittstellen, -organisation und -ablauf sowie gesellschaftliche/soziale Faktoren sind die Quellen sowohl der psychischen Belastungsfaktoren als auch der äußeren Ressourcen. Sie sind daher im Sinne des S-O-RModells (Abschn. 1.1.1) als Stimuli aufzufassen und damit auf der Einwirkungsseite.

Zwischen Ressourcen und Belastungsfaktoren herrscht eine Interdependenz. Je nach Vorhandensein oder NichtVorhandensein und bezogen auf Bedarf oder keinen Bedarf können Ressourcen selbst Belastungsfaktoren sein (verdeutlicht im Arbeitsmodell durch den Doppelpfeil). Wenn beispielsweise der psychische Belastungsfaktor ,Zeitvorgabe" in der menschlichen Zielpopulation zu einem Engpass führt, kann die Ressource „Hilfskraft“ den Engpass verringern. Somit moderiert die Ressource zwischen Engpass und Belastungsfaktor. Andererseits moderieren auch die
Belastungsfaktoren die Ressourcen. Beispielsweise kann die Zeitvorgabe dazu führen, dass sich Beschäftigte um eine mögliche Ressource (hier Hilfskraft) überhaupt erst bemühen.

Die psychische Belastung kann gemäß der Definition der DIN EN ISO 10075-1 zu einem Engpass führen (siehe Abschn. 1.1.5), der im Bereich der kognitiven und/oder emotionalen Verarbeitungskapazität liegt. Ein solcher Engpass kann beim Annähern an die Grenze der Verarbeitungskapazität zu einer Fehlbeanspruchung und damit zu einer psychischen Gefährdung und aversiven Folgen führen.

Das Arbeitsmodell zeigt ebenfalls die (definitionsgemäß neutrale) psychische Beanspruchung, die das Resultat der Interaktion zwischen psychischer Belastung und Mensch ist und in Beanspruchungsfolgen resultiert. Diese können sowohl positiv (z. B. Übung) als auch negativ (z. B. permanente Stressreaktion) sein.

Obwohl sich die Forschungsarbeit auf die im Modell linke, grau hinterlegte Belastungsseite fokussiert (Fehlbelastung und Gefährdung), kann die Gefährdung nur durch aversive Beanspruchungsfolgen bei der Betrachtung der jeweiligen menschlichen Zielpopulation erfolgen.

Wenn die Folgen der Einwirkung aversiv bzw. beeinträchtigend sind, dann liegt eine Fehlbelastung vor. Diese definiert sich über die beeinträchtigenden Konsequenzen der Belastungseinwirkung und der daraus folgenden Prozesse. Engpässe führen zur Fehlbeanspruchung und darüber zu aversiven Folgen, Scheitern oder Rückwirkung, die weitere, auch über die handelnde Person hinausgehende Folgen generieren können.

Betrachtet man das Arbeitsmodell aus psychologischer Perspektive, so wandeln sich die Fachdisziplinen von links nach rechts. Der dunkelgraue Teil umfasst die allgemeinpsychologische Betrachtungsweise. Die allgemeine Psychologie befasst sich mit psychischen Funktionen, denen nahezu alle Menschen unterliegen. Der Bereich, der auf die individuellen Unterschiede von Menschen ausgerichtet ist (mittlere und rechte Spalte des Modells) umfasst die Differentielle Psychologie (Asendorpf 2009). Hier geht es darum, die interindividuellen Unterschiede (die letztendlich zur individuellen unterschiedlichen psychischen Beanspruchung führen) offenzulegen. Der differentielle Bereich des Arbeitsmodells wird in dieser Forschungsarbeit nicht auf ein Individuum ausgerichtet, sondern auf eine prospektive Zielpopulation von Beschäftigten (vgl. Abschn. 1.1.7). Es geht in dieser Betrachtungsweise nicht um individuelle, sondern um kollektive, möglichst allgemeingültige Grenzwerte für eine Zielpopulation. Der Grund liegt im Arbeitsschutzgesetz, welches explizit die Betrachtung der Belastung fordert, nicht die der Beanspruchung (vermutlich ist es redundant $\mathrm{zu}$ wiederholen, dass Beanspruchungsfolgen als Indikatoren der Belastung herangezogen werden müssen). Es ist bei den folgenden Ausführungen und Über- 
legungen essenziell, sich dieser Beschränkung des Modells bewusst zu sein, denn viel zu oft gibt es in der Literatur unsaubere Trennungen zwischen Belastung, Beanspruchung und deren Folgen. Der Übergang zwischen diesen Betrachtungsweisen ist im Modell bewusst gewählt, denn der Mensch ist einerseits das Maß für die Grenzwerte im Sinne der Allgemeinpsychologie (oder in arbeitswissenschaftlicher Terminologie im Sinne der Arbeitsgestaltung) durch Engpässe im Leistungsvermögen und andererseits auch Fokus der Beanspruchungsfolgen im Sinne der Differentiellen Psychologie. Der Übergang ist fließend, denn braucht man die Auswirkungen im Menschen um Grenzwerte psychischer Belastung festlegen zu können (woran sonst sollte man diese festmachen), ist der Mensch das wichtigste Element.

Somit gibt es auch in diesem Arbeitsmodell eine gewisse Unmöglichkeit der Trennung, denn wenn es um die Aufrechterhaltung von Schädigungslosigkeit geht, kann diese zwar theoretisch über Belastungsfolgen diskutiert werden, aversive Folgen oder gar eine Schädigung werden sich jedoch immer durch Auswirkungen am oder im Menschen zeigen.

Mit anderen Worten trägt das Arbeitsmodell sowohl dem Arbeitsschutzgesetz Rechnung, das sich auf psychische Belastung bezieht, als auch der DIN, die besagt, dass es psychische Belastung gibt, die menschliche Kapazitäten, z.B. das menschliche Vermögen zur Informationsverarbeitung, auf der Eingangs- wie auf der Ausgangsseite, übersteigt. Dies zeigt die Notwendigkeit, sich ausschließlich auf Belastung zu beziehen. Diese stößt auf einen menschlichen Engpass (nämlich die natürliche Verarbeitungskapazität) und dieses Zusammenspiel beeinflusst, ob die Belastung dazu führt, dass die Beanspruchungsfolgen förderlich oder beeinträchtigend sind. Wenn Belastungsfaktoren und Ressourcen für eine Zielpopulation derart gestaltet sind, dass die resultierende psychische Belastung NICHT die Verarbeitungskapazität übersteigt, wird es nicht zu einer Fehlbelastung und damit nicht zu einer Gefährdung kommen. Und das passiert alles auf der Belastungsseite. Selbstverständlich muss das am Menschen untersucht werden, denn nur hier kann eine Bestimmung der Informationsverarbeitungskapazität herkommen - im Sinne der Allgemeinen Psychologie.

Das Arbeitsmodell dient der Untersuchung der verschiedenen Einwirkfaktoren und deren Auswirkungen, um über beeinträchtigende Beanspruchungsfolgen und dadurch entstehende aversive Belastungsfolgen auf die Gefährdung und damit die Fehlbelastung rückschließen zu können. Zahlreiche Rückkopplungsprozesse sind in diesem Modell denkbar und können durch Forschung empirisch ergänzt werden. Das Arbeitsmodell gibt hierfür einen grundlegenden Rahmen vor.

\section{2 Überblick über den Stand der Forschung}

Gefordert wird vom Arbeitsschutzgesetz die Erfassung der psychischen Belastung für jeden Arbeitsplatz. Jedoch liefert der Gesetzgeber keine konkreten Durchführungsvorschriften zur Umsetzung dieser Forderung, weder in Bezug auf den Erfassungsgegenstand noch auf die Methode der Erfassung oder die Beurteilung der Ergebnisse. Aufgrund zahlreicher unscharfer Konzeptualisierungen des Messgegenstandes und zusätzlich unterschiedlicher Erhebungsverfahren und Vorgehensweisen kommt es innerhalb der Betriebe zu Unsicherheiten (Bamberg und Mohr 2016; Schuller 2020). Dass es keine einheitlichen Durchführungsvorschriften gibt, hat auch der deutsche Bundesrat festgestellt (Bundesrat Drucksache 315/13 2014, § 4 Abs. 1 S. 1). Die vorhandenen Erhebungsinstrumente vergleichen bestenfalls analysierte Werte der vorhandenen Belastungsfaktoren oder Beanspruchungsfolgen mit Durchschnittswerten in anderen Betrieben oder Abteilungen (Richter und Kuhn 2010). Die Sinnhaftigkeit dieses Vorgehens ist zumindest diskussionswürdig. Eine festgestellte Belastung ist nicht zwingend eine Gefährdung (Paridon und Mühlbach 2016). Erst die Belastungsfolgen machen Belastung zur Gefährdung, denn Belastung ist definitionsgemäß neutral.

Aufgrund dieser vorliegenden Diskrepanz sollen nach $\S 20 \mathrm{a}$ ArbSchG Bund, Länder und Unfallversicherungsträger einer gemeinsamen Arbeitsschutzstrategie folgen und für deren Umsetzung und Aktualität Sorge tragen (Richardi 2017). Zur Sicherstellung steht die Gemeinsame Deutsche Arbeitsschutzstrategie (GDA) zur Verfügung, die auf $\S 20 \mathrm{a}$ ArbSchG basiert und für ein vereinheitlichtes Vorgehen und Transparenz bei Gefährdungsbeurteilungen sorgen soll. Dies ist ein ausgesprochen sinnvolles Vorgehen. Bedauerlicherweise wird außer Acht gelassen, dass alle - nicht nur vorab als relevant eingeschätzte und deshalb erhobene - Gefährdungen zu beurteilen sind. Wie der Weg von der (neutralen!) Belastung zur Gefährdung stattfindet, wird nicht ausreichend erläutert.

Eine abschließende Beurteilung der Gefährdung aufgrund der Arbeitsbedingungen ist trotz aller Fortschritte und Bemühungen bisher nur im Einzelfall möglich (beispielsweise lange Arbeitszeiten ohne Pause). Ein Grund liegt, wie bereits erwähnt, in uneinheitlichen Verständnissen und Terminologien psychischer Belastung. Ein weiteres Hemmnis stellen die schwer fassbaren Ketten von Wirkzusammenhängen dar, denn die unterschiedlichen Belastungsfaktoren aus der Arbeit interagieren miteinander in Abhängigkeit ihrer Intensität und Extensität. Zudem hängt psychische Belastung primär mit kontrollierter und automatisierter Informationsverarbeitung zusammen, was sich als eher schwierig zu erforschendes Feld darstellt (DIN EN ISO 10075-1 2018) und im Zuge der technischen Automatisierung von Arbeitsprozessen noch verschärfend wirkt 
Tab. 1 Etablierte wissenschaftliche Anforderungen der Bewertung und Gestaltung von Arbeit. (Basierend auf Rohmert 1972; Hacker 1984) Table 1 Established scientific requirements of work evaluation and design. (Based on Rohmert 1972; Hacker 1984)

\begin{tabular}{lll}
\hline Kriterien & Anforderungen & Bewertungskriterien \\
\hline Ausführbarkeit & $\begin{array}{l}\text { Tätigkeitsanforderungen müssen auf Dauer anforderungs- } \\
\text { gerecht erfüllbar sein }\end{array}$ & $\begin{array}{l}\text { Ergonomische Normwerte (anthropometrische oder } \\
\text { sinnesphysiologische Normen) }\end{array}$ \\
$\begin{array}{l}\text { Erträglichkeit/ } \\
\text { Schädigungslosigkeit }\end{array}$ & $\begin{array}{l}\text { Tätigkeit muss dauerhaft geleistet werden können, ohne } \\
\text { dass körperliche oder geistige Schäden auftreten }\end{array}$ & $\begin{array}{l}\text { Arbeitsmedizinische Fragestellung, wie z. B. } \\
\text { MAK- oder BAT-Werte, Erkrankungs- und Un- } \\
\text { fallstatistiken } \\
\text { Erhaltung von Fähigkeiten, Fertigkeiten und Wis- } \\
\text { sen }\end{array}$ \\
$\begin{array}{l}\text { Zumutbarkeit }{ }^{\mathrm{a}} / \\
\text { Beeinträchtigungsfreiheit }\end{array}$ & $\begin{array}{l}\text { Negative Beanspruchungsfolgen müssen reversibel sein } \\
\text { (d.h. durch Erholung kompensiert werden), zumutbare } \\
\text { Beanspruchung }\end{array}$ & $\begin{array}{l}\text { Maße der Befindensbeeinträchtigung oder psycho- } \\
\text { physiologische Kennwerte, z. B. Ermüdung und } \\
\text { Persönlichkeits-/Lern-/ } \\
\text { Gesundheitsförderlichkeit } \\
\text { und Zufriedenheit }\end{array}$ \\
$\begin{array}{l}\text { Tätigkeit muss Ausbau von Fähigkeiten und Fertigkeiten } \\
\text { ermöglichen. Körperliches, geistiges und soziales Wohl- } \\
\text { befinden muss auch bei sich ändernden Anforderungen } \\
\text { gesichert sein }\end{array}$ & $\begin{array}{l}\text { Weiterentwicklung von Fähigkeiten, Fertigkeiten } \\
\text { und Wissen }\end{array}$ \\
\hline
\end{tabular}

${ }^{a}$ Verhältnismäßigkeit der wirtschaftlichen und humanen Interessen

bIm Sinne einer vorwiegend positiven emotionalen Verfassung

(Jipp und Steil 2021). Ein weiterer Grund liegt im Mangel von wissenschaftlich fundierten Grenzwerten psychischer Belastung.

Nicht nur das Arbeitsschutzgesetz ( $(2)$, sondern auch Betriebsverfassungsgesetz ( $\S \S 90,91$ ), Arbeitszeitgesetz (§ 6) sowie Jugendarbeitsschutzgesetz (§ 28) fordern die Bewertung und Gestaltung menschengerechter Arbeit gemäß etablierten wissenschaftlichen Erkenntnissen. Einen ersten Ansatz hierzu stellt Rohmert (1972, S. 8) mit den vier Bewertungsebenen Ausführbarkeit (z.B. maximaler Greifraum), Erträglichkeit (z. B. Grenzen der Ausdauer bei Muskelarbeit), Zumutbarkeit (Akzeptanz der Bedingungen durch [nicht näher definierte] Gruppen) sowie Zufriedenheit (Akzeptanz basierend auf individueller Zufriedenheit) auf. Hacker (1984, S. 30) erweitert diese ingenieurwissenschaftlichen Defizitkriterien inhaltlich und fügt das (arbeits)psychologische Wachstumskriterium Persönlichkeitsförderlichkeit hinzu. Sein Vorschlag lautet: Ausführbarkeit (z. B. anthropometrische Normen), Schädigungslosigkeit (z. B. MAK-Werte), Beeinträchtigungsfreiheit (z.B. Befindensbeeinträchtigungen) sowie Persönlichkeitsförderlichkeit (z.B. erforderliche Lernaktivitäten). Die formulierten Kriterien werden bis heute den wissenschaftlichen Erkenntnissen und veränderten Arbeitsbedingungen angepasst (beispielsweise Hacker 1986; Richter und Hacker 1998; Ulich 2005; REFA-Institut 2016).

Momentan finden als (geforderte) etablierte wissenschaftliche Anforderungen der Bewertung und Gestaltung von Arbeit die in Tab. 1 dargestellten Kriterien Verwendung.

Besonders wichtig für die vorliegende Forschungsarbeit ist die Frage nach der Erträglichkeit bzw. Schädigungslosigkeit von Belastung, denn diese Gestaltungsanforderungen verlangen, dass eine Belastung aus der Arbeitsumwelt nur so stark ausgebildet sein darf, dass diese ohne gesund- heitliche Schäden für eine ganze Schicht (in der Regel acht Stunden täglich) über ein ganzes Arbeitsleben ertragen werden kann. Um dies sicherzustellen, gibt es im Bereich der körperlichen Belastung Grenzwerte für verschiedene $\mathrm{Ar}$ ten der muskulären oder kardiopulmonalen Belastung, wie beispielsweise die Leitmerkmalmethode oder MAK-Werte (REFA-Institut 2016; BAuA 2017). Die Dauerleistungsgrenzen werden auf Basis von Beanspruchungsparametern festgelegt (beispielsweise für dynamische Muskelarbeit auf Basis der Herzschlagfrequenz).

Um die geforderte Erträglichkeit psychischer Belastung sicherstellen zu können, ist zunächst zu prüfen, ob und inwieweit aktuell Grenzwerte für psychische Belastung und die dazugehörigen Belastungsfaktoren vorliegen, anhand derer Gefährdungsbeurteilungen konkret umzusetzen wären.

Die Bundesanstalt für Arbeitsschutz und Arbeitsmedizin (BAuA 2017) weist in ihrer wissenschaftlichen Standortbestimmung darauf hin, dass bisher bei physischer Belastung normative Schwellenwerte vorliegen (hierzu zählen beispielsweise Lärm- und Gefahrstoffexposition). Zudem ergeben sich aus juristischen Gründen Belastungsbeschränkungen, hier wäre zum Beispiel das ArbSchG zu nennen, welches Rahmen und Dauer der zulässigen Arbeitszeit definiert. Trotzdem gilt für die meisten psychischen Belastungsfaktoren, dass keine quantitativ eindeutig definierten Grenzwerte zur Verfügung stehen. Die Forschung zur Quantifizierung von Arbeitsbelastung hat zwar hoch belasteten Mitarbeitenden mehr Pausen, Urlaub und Kuren verschafft, die Bestimmung eines sicheren Grenzwertes für zu wenig oder zu viel Belastung ist jedoch bisher nicht gelungen (z. B. bei Flugsicherungspersonal Vogt et al. 2006).

Die Deutsche Gesetzliche Unfallversicherung (DGUV 2013) kommt zur Auffassung, dass es keine Grenzwerte für psychische Belastung gibt. Argumentiert wird, dass ein 
einzelner Belastungsfaktor nicht zwingend schädlich ist, sondern erst die Überlagerung mehrerer Belastungsfaktoren (DGUV 2013). Somit gibt es nahezu unzählig viele Kombinationsmöglichkeiten von Belastungsfaktoren, die als Variablen in die Grenzwertbestimmung eingehen müssen. Jedoch ist dies bei physischer Grenzwertbestimmung nicht anders. Auch die BAuA (2017) beschreibt zahlreiche Herausforderungen bei der Bestimmung von Grenzwerten. So wird darauf hingewiesen, dass Arbeitsbedingungsfaktoren von betrieblichen Aushandlungsprozessen, sozialen Interaktionen und spezifischen arbeitsorganisatorischen Lösungen bestimmt sind, was eine generalisierte Messung und Definition verkompliziert (BAuA 2017). Jedoch ist ein Summenmaß nicht angedacht, denn auch bei physischen Grenzwerten existieren solche Summenmaße nicht. Ebenso ergänzt die BAuA, dass auch Ressourcen wichtige Elemente bei der Grenzwertbestimmung sind. Diese greift das Arbeitsmodell (siehe Abb. 1, (8)) im Sinne von kollektiven Ressourcen zur Erreichung von kollektivem Arbeitsschutz auf. Es lässt sich festhalten, dass hinsichtlich der verschiedenen psychischen Arbeitsbedingungsfaktoren keine eindeutig quantitativ definierten Grenzwerte zur Verfügung stehen (BAuA 2017).

Einige, vor allen Dingen arbeitspsychologische Modelle, thematisieren ebenfalls das Zusammenspiel von Belastung, Beanspruchung und Ressourcen am Arbeitsplatz. Beispiele sind:

- Stressmodell nach McGrath (1981)

- Transaktionales Stressmodell (Lazarus und Folkman 1984)

- Theorie der Ressourcenerhaltung (Hobfoll und Buchwald 2004)

- Job-Demands-Resources Modell (Karasek 1979; Bakker und Demerouti 2007).

Vereinfacht dargestellt können in diesen Modellen Ressourcen Belastungsfolgen beeinflussen und auch optimieren.

\subsection{Forschungsfragen}

Die übergeordnete Forschungsfrage des gesamten Forschungsvorhabens lautet: Können Grenzwerte psychischer Belastung eindeutig festgelegt werden?

Neben der Entwicklung eines Arbeitsmodells zur Beantwortung dieser Frage trägt dieser Beitrag dazu bei, ein Kategoriensystem zur Erhebung von Belastungsfaktoren und Ressourcen zu entwickeln. Daher wird die folgende Forschungsfrage gestellt:

Gibt es ein berufsgruppenübergreifendes Kategoriensystem, um Belastungsfaktoren und Ressourcen zu erheben und damit statistischen Modellen zugänglich zu machen?

\section{Methoden}

Die Methodik basiert auf einer hermeneutisch-interpretativen Vorgehensweise. Im Rahmen der qualitativen Forschungsmethodik orientiert sich die Vorgehensweise hauptsächlich an Kuckartz (2018) und hier im Speziellen an der inhaltlich strukturierenden qualitativen Inhaltsanalyse sowie der evaluativen qualitativen Inhaltsanalyse.

Die Vorgehensweise zur Beantwortung der Forschungsfrage ist in Tab. 2 dargestellt.

\subsection{Datenerhebung}

Insgesamt werden 10 Samplings herangezogen, um die Forschungsfrage zu beantworten. Die Erhebungen haben zwischen Februar 2019 und März 2021 stattgefunden. Die Fallauswahl wird top down getroffen auf Basis von Literaturstudien zu Belastungsfaktoren. Die ersten neun Samplings werden aus acht möglichst heterogenen Berufsgruppen gezogen (siehe Tab. 3). Lehrende werden mit zwei Samplings erhoben, da nach dem ersten Sampling ungeplant ein Schulprojektauftrag erfolgt und die Möglichkeit zur Validierung ergriffen wird. Alle Samplings der Interviews werden in Unternehmen im Rhein-Main-Gebiet von geschulten Interviewenden basierend auf vorhandenen Berufskontakten gezogen.

Die ersten neun Samplings dienen folgenden Zwecken:

- Feststellung von branchenspezifischen Belastungsfaktoren (vgl. Abschn. 1.1.1) sowie möglichen Ressourcen

- Sammlung von Praxisbeispielen für die einzelnen Felder der Kategoriensysteme

- Optimierung der Interviewleitfäden

- Identifikation von branchenübergreifenden Belastungsfaktoren sowie möglicher Ressourcen

Um sicherzustellen, dass ausreichend Nennungen der berufsspezifischen und berufsübergreifenden Belastungsfaktoren und Ressourcen erfolgen, wird festgelegt, dass pro Berufsgruppe mindestens sieben Interviews geführt werden. Weitere Interviews sollen erfolgen, wenn davon ausgegangen wird, dass diese zu zusätzlichen Informationen führen.

Es handelt sich hierbei um ein iteratives Vorgehen, welches die Erkenntnisse aus den vorherigen Samplings im Forschungsprozess umsetzt. Auf diese Weise können die Kategoriensysteme ${ }^{4}$ schrittweise verbessert und durch Subkategorien erweitert werden. Die Inhaltsanalysen der Transkripte der Interviews werden sowohl induktiv als auch deduktiv ausgewertet. Die deduktive Analyse erfolgt basierend auf der analysierten Literatur (siehe Abschn. 1.1.6),

\footnotetext{
${ }^{4}$ Eines für Belastungsfaktoren, ein anderes für Ressourcen, basierend auf den gleichen Hauptkategorien (vgl. Abschn. 1.1.6 und (9) in Abb. 1).
} 
Tab. 2 Forschungsphasen der Inhaltsanalysen

Table 2 Research phases of the content analyses

\begin{tabular}{|c|c|c|}
\hline Phase & $\begin{array}{l}\text { Theoretischer } \\
\text { Bezug }\end{array}$ & Bezug zur Forschungsfrage \\
\hline $\begin{array}{l}\text { Festlegung der Bewertungskate- } \\
\text { gorien }\end{array}$ & $\begin{array}{l}\text { Evaluative } \\
\text { qualitative } \\
\text { Inhaltsanalyse }\end{array}$ & $\begin{array}{l}\text { Die Herleitung der Bewertungskategorien wird in Abschn. 1.1.6 Arbeitsbedingungen } \\
\text { beschrieben }\end{array}$ \\
\hline $\begin{array}{l}\text { Identifizieren und codieren der } \\
\text { für die Bewertungskategorie } \\
\text { relevanten Textstellen }\end{array}$ & $\begin{array}{l}\text { Evaluative } \\
\text { qualitative } \\
\text { Inhaltsanalyse }\end{array}$ & $\begin{array}{l}\text { In einem iterativen Prozess werden die transkribierten Interviews durchgearbeitet und } \\
\text { falls möglich den vorhandenen Kategorien zugeordnet }\end{array}$ \\
\hline $\begin{array}{l}\text { Codierte Segmente der Bewer- } \\
\text { tungskategorie fallbezogen zu- } \\
\text { sammenstellen }\end{array}$ & $\begin{array}{l}\text { Evaluative } \\
\text { qualitative } \\
\text { Inhaltsanalyse }\end{array}$ & $\begin{array}{l}\text { Alle in eine Kategorie fallende Äußerungen werden zusammengefasst - diese erwei- } \\
\text { tern sich nach jeder Interviewreihe (vgl. Tab. 3) }\end{array}$ \\
\hline $\begin{array}{l}\text { Codieren des gesamten Materials } \\
\text { mit den Hauptkategorien }\end{array}$ & $\begin{array}{l}\text { Inhaltlich struk- } \\
\text { turierende In- } \\
\text { haltsanalyse }\end{array}$ & $\begin{array}{l}\text { Herausarbeitung der Textstellen, die nicht dem vorhandenen Kategoriensystem zuzu- } \\
\text { ordnen sind. Überschneidungen zwischen den Kategorien werden aufgezeigt }\end{array}$ \\
\hline $\begin{array}{l}\text { Zusammenstellen aller mit der } \\
\text { gleichen Hauptkategorie codier- } \\
\text { ten Textstellen }\end{array}$ & $\begin{array}{l}\text { Inhaltlich struk- } \\
\text { turierende In- } \\
\text { haltsanalyse }\end{array}$ & $\begin{array}{l}\text { Der iterative Prozess, der eine Überprüfung der Zuordnung und des Kategoriensys- } \\
\text { tems nach jeder Interviewreihe vorsieht, ermöglicht die permanente Spezifizierung der } \\
\text { Kategorien }\end{array}$ \\
\hline $\begin{array}{l}\text { Induktives Bestimmen von Sub- } \\
\text { kategorien am Material }\end{array}$ & $\begin{array}{l}\text { Inhaltlich struk- } \\
\text { turierende In- } \\
\text { haltsanalyse }\end{array}$ & $\begin{array}{l}\text { Die Subkategorien werden detaillierter und ausdifferenzierter. Deren Überprüfung } \\
\text { erfolgt ebenfalls iterativ }\end{array}$ \\
\hline $\begin{array}{l}\text { Codieren des kompletten Mate- } \\
\text { rials mit dem ausdifferenzierten } \\
\text { Kategoriensystem }\end{array}$ & $\begin{array}{l}\text { Inhaltlich struk- } \\
\text { turierende In- } \\
\text { haltsanalyse }\end{array}$ & $\begin{array}{l}\text { Dieser Schritt wird mittels des zehnten Samplings (Tab. 3) vorgenommen und ist dazu } \\
\text { vorgesehen, die Kategorien abermals zu validieren }\end{array}$ \\
\hline $\begin{array}{l}\text { Erstellung einer abschließenden } \\
\text { Kategoriendefinition }\end{array}$ & - & $\begin{array}{l}\text { Basierend auf den vorherigen Forschungsphasen werden die Kategorien inhaltlich be- } \\
\text { schrieben und klare Regeln entwickelt, wann eine Aussage der Versuchspersonen in } \\
\text { welche Kategorie einzuordnen ist. Hierbei werden zahlreiche Beispiele aus den In- } \\
\text { terviews benannt. Weitere Beispiele, die aus der Literatur oder aber dem logischen } \\
\text { Sachverstand entnommen werden, ergänzen die Beschreibungen. Zudem finden Ab- } \\
\text { grenzungen der Kategorien statt }\end{array}$ \\
\hline
\end{tabular}

Tab. 3 Gezogene Samplings mit Anzahl $(N)$ und Zeitpunkt $(t)$ der Erhebung

Table 3 Samplings with number $(N)$ and time $(t)$ of survey

\begin{tabular}{lll}
\hline Sampling & $N$ & $\mathrm{t}$ \\
\hline Pflegepersonal & 7 & $02 / 2019$ \\
Lehrende (erstes Sampling) & 7 & $03 / 2019$ \\
Wissenschaftliche Mitarbeitende & 7 & $04 / 2019$ \\
Gastgewerbe & 7 & $06 / 2019$ \\
IT-Beschäftigte & 7 & $08 / 2019$ \\
Unternehmensberatende & $10 / 2019$ \\
Recruiter/-innen & 7 & $72 / 2019$ \\
Produktionsarbeitende im Schichtdienst & $02 / 2020$ \\
Lehrende (zweites Sampling) & $06 / 2020$ \\
Gemischtes Sampling (Kaufmännische Angestellte, Pflegedienstleitung, Sachbearbeitung, Personalleitung, Finanz- & 15 & $03 / 2021$ \\
analyse, Maschinenbau-Ingenieurwesen, Vermögensberatung, Dolmetscher/-innen, Kassierer/-innen, Kundenser- & 7 \\
vicemitarbeitende, Praxisassistenz, Lehrkraft, Erzieher/-in, Tierarzt/Tierärztin, Fachangestellte Gesundheitswesen) & \\
\hline
\end{tabular}

die induktive Analyse überprüft und erweitert die Kategoriensysteme und deren Subkategorien basierend auf neuen Erkenntnissen aus den Interviews.

Das zehnte Sampling verfolgt folgende Ziele:

- Überprüfung, ob die Kategoriensysteme für weitere Berufsgruppen verwendet werden können

- Erweiterung der Praxisbeispiele für die einzelnen Felder der Kategoriensysteme
Bei diesem Sampling gibt es keine Setzung einer Mindestanzahl von Interviews. Nach 15 Interviews sind alle formulierten Ziele zum momentanen Stand der Forschungsarbeit erreicht.

\subsection{Interviewleitfaden}

Alle Interviews folgen dem gleichen halbstrukturierten Interviewleitfaden, der durch kleinere Modifikationen an die 
Berufsgruppen angepasst wird. Die Auswahl der Interviewten erfolgt zufällig und bedarf der Freiwilligkeit der Befragten. Alle Interviews werden digital aufgezeichnet. Die Auswertung erfolgt durch (a) Transkription (b) Verstichwortung und (c) Kategorienbildung. Die Interviews fokussieren die Art der auftretenden Belastungsfaktoren sowie das Vorhandensein und der Wirkung von Ressourcen.

In einer Vorstudie wird der Interviewleitfaden hinsichtlich des Verständnisses an fünf Versuchspersonen überprüft. Dies gibt beispielsweise Hinweise auf Nachfragen, die gestellt werden können, wenn die erwartete oder benötigte Antwort nicht erfolgt. Da die Interviews in jeder Berufsgruppe von unterschiedlichen Interviewenden geführt werden, ist es notwendig, diese zu schulen und ihnen im Interviewleitfaden ausreichend Handlungsanweisungen $\mathrm{zu}$ geben. Der Interviewleitfaden enthält somit neben den Fragen auch Hintergrundinformationen, Gesprächstext als ergänzende Nachfragen sowie bei Bedarf Handlungsanweisungen.

Die Interviews beginnen mit einem Dank an die Teilnehmenden, der Zusicherung einer vollkommen anonymen Weiterverarbeitung der Daten sowie der Bitte um Erlaubnis, das Gespräch digital aufzeichnen zu dürfen. Die Versuchsleitenden erhalten die Anweisung, bei zu wenig Details in der Antwort mit beispielsweise ,was noch?“ nachzufragen oder aber eine kurze Pause im Gespräch auszuhalten und auf weitere Informationen zu warten.

Die erste Frage des Interviewleitfadens lautet „Wenn jemand, der Sie nicht kennt, Sie fragen würde, was Sie beruflich machen, wie würden Sie Ihre Tätigkeit beschreiben?". Hintergrund dieser Frage ist, eine Tätigkeitsbeschreibung zu erhalten, um einer Berufsgruppe bestimmte Belastungsfaktoren zuordnen zu können. Da das Wort „Belastung“ umgangssprachlich negativ konnotiert ist, wird die Verwendung im Interview vermieden. Die Vorstudie zeigt, dass Interviewte hier lediglich mit ihrer Berufsbezeichnung antworten, beispielsweise „Patienten betreuen“, „Computer instandsetzen“ oder „Lehre“. Bei solchen Antworten sind die Versuchsleitenden angehalten, nach einem typischen Arbeitstag zu fragen.

Die zweite Frage des Interviewleitfadens bezieht sich auf häufiger auftretende Belastungsfaktoren. Auch hier wird der negativ behaftete Begriff „Belastung“ vermieden und durch Anforderungen ersetzt: „Wenn Sie an Ihre Tätigkeit denken, welche hauptsächlichen Anforderungen haben Sie zu erfüllen? “. Die Vorstudie verdeutlicht, dass Nachfragen notwendig sein können, weshalb die Versuchsleitenden Anregungen zum Nachfragen erhalten, wie ,müssen Sie zum Beispiel pünktlich sein oder ist die Kundschaft sehr fordernd oder müssen Sie oft mit Ihren Vorgesetzten oder Ihren Teammitgliedern zusammenarbeiten, müssen Sie häufig Schutzkleidung tragen“.
Da Belastung bislang gemäß Definition neutral betrachtet wird, das Interview jedoch auch Fehlbelastung erfassen soll, richtet sich die dritte Interviewfrage auf Belastungsfaktoren, die negativ sind (bezogen auf ihre Auswirkungen). Um auch hier ein allgemeines Verständnis zu schaffen, werden negative Belastungsfaktoren als ,,anstrengend“ oder „,nervig“ bezeichnet: „Wenn Sie sich Ihre Arbeit alles in allem einmal betrachten, also zum Beispiel Ihr Verhältnis zu Ihren Teammitgliedern oder Vorgesetzten, oder Ihre Arbeitszeiten, oder Ihre Arbeitsmittel, oder Ihre Bezahlung, oder Ihren Tätigkeitsbereich ... welche Probleme sind für Sie am anstrengendsten oder nervigsten?". Auch hier zeigt die Vorstudie, dass Nachfragen notwendig sein können, wenn beispielsweise die Antwort ,alle gleich“ erfolgt. Die Versuchsleitenden bekommen den Hinweis nachzufragen „Aber es gibt doch immer etwas, das einen am meisten nervt oder anstrengt."

Die darauffolgende vierte Interviewfrage leitet zu möglicher Fehlbelastung über und greift die Belastungsfaktoren mit negativen Folgen aus Interviewfrage drei auf, die die Befragten benennen: „Beschreiben Sie eine Situation, in der dieses Problem alleine oder mit anderen Problemen zusammen Sie überlastet oder gestresst hat." Werden in der vorherigen Frage mehrere negative Belastungsfaktoren angesprochen, dann lautet die Frage: „Beschreiben Sie Situationen, in denen diese Probleme alleine oder mit anderen Problemen zusammen Sie überlastet oder gestresst haben." Die Versuchsleitenden sind angehalten darauf $\mathrm{zu}$ achten, dass die Befragten beschreiben, wann welche negative $\mathrm{Be}$ lastungsfolge zu einer Fehlbelastung führt. Besonders ist die Aufmerksamkeit der Versuchsleitenden darauf zu richten, die Antworten für die fünfte Interviewfrage präsent zu haben, um sie wieder aufgreifen zu können: „Sie haben mir folgende Probleme als manchmal überlastend und stressig berichtet (auflisten)." Wenn nur ein Belastungsfaktor aufgeführt wird dann: „Sie haben mir XXXX als manchmal überlastend und stressig berichtet". Der weitere Wortlaut der Frage führt dazu, mögliche Ressourcen für die entsprechende Fehlbelastung zu eruieren: „Was könnte Ihnen helfen, diese Anforderungen zu reduzieren? Was hat Ihnen bereits einmal geholfen, diese Anforderungen zu reduzieren? Was war an diesen Tagen anders?" Die Versuchsleitenden können Unterstützung bei den Antworten anbieten durch Nachfragen wie „Gibt es irgendetwas, das an Ihrem Arbeitsplatz verändert werden könnte?“ oder „Bräuchten Sie mehr Unterstützung (wenn ja, von wem) ${ }^{5}$ ?“ oder „Fehlen Ihnen bestimmte Kenntnisse oder Fähigkeiten?“.

Die sechste und letzte Interviewfrage erläutert den Befragten den Zweck des Interviews und ermöglicht es diesen noch Ergänzungen anzubringen. In dieser letzten Frage wird der Begriff „Belastung“ bewusst verwendet, um eventuell

\footnotetext{
5 Im Sinne von fehlenden Ressourcen.
} 
noch neue Assoziationen hervorzurufen: „Unser Ziel war es mit diesem Interview herauszubekommen, welche Belastung hauptsächlich im (Berufsgruppe nennen) Bereich vorkommen und mit welchen Möglichkeiten diese reduziert werden können. Wenn Sie noch einmal über Ihre gesamte Arbeitssituation nachdenken, gibt es noch mehr Belastungsfaktoren, die Sie oder Kolleginnen und Kollegen von Ihnen hier anführen könnten?"“

Das Interview schließt mit einem Dank an die Teilnehmenden.

\subsection{Expert/-innengruppe}

Neben den qualitativen Erhebungen und deren Auswertungen bedarf es einer Expert/-innengruppe, die folgende Ziele verfolgt:

a) Entwicklung der noch nicht vorhandenen Definitionen

b) Analyse des Datenmaterials hinsichtlich Belastungsfaktoren und Ressourcen, die einerseits erstens global sind und damit alle untersuchten Berufsgruppen betreffen und die zweitens von einer Organisation beeinflusst und kontrolliert werden können

c) Erstellung zweier übergeordneter Kategoriensysteme für Belastungsfaktoren sowie Ressourcen, die einerseits standardisiert sind und andererseits offen und erweiterbar für Kategorien, die im analysierten Datenmaterial noch nicht identifiziert werden.

Die Expert/-innengruppe setzt sich aus teilweise wechselnden Mitgliedern zusammen. Hierzu gehören dauerhaft die Autorenschaft dieses Beitrages sowie wechselnd fünf weitere Professor/-innen der Psychologie und Ingenieurswissenschaften sowie studentische Projektgruppen. Der Forschungsprozess der Expert/-innengruppe verpflichtet sich dem Prinzip der hermeneutisch-interpretativen Vorgehensweise.

\subsection{Gütekriterien}

Zur Sicherstellung der internen Studiengüte erfolgen Audioaufnahmen von allen Interviews und der gesamte Forschungsprozess wird fortlaufend dokumentiert. Die Interviews werden vollständig transkribiert nach den Transkriptionsregeln angelehnt an Kuckartz (2018). Die Transkription wird von den Interviewenden vorgenommen und von einer nicht in diese Interviewreihe involvierte Person der Expert/-innengruppe überprüft. Alle Daten sind anonymisiert weiterverarbeitet. Die weitere Datenanalyse wird computergestützt übernommen und von mindestens zwei Codierenden getrennt voneinander mit einem abschließenden Vergleich überprüft. Abweichende Fälle werden genutzt, um das Kategoriensystem zu erweitern. Die einzelnen Katego- rien sowie deren Subkategorien sind präzise definiert und mit Zitaten aus den Interviews belegt.

Die Intercoder-Objektivität wird durch mehrere Codierende für das gleiche Material sichergestellt. Die Intracoder-Reliabilität wird durch mehrfache Überprüfung der Codierungen nach weiterem Erkenntnisgewinn durch die gleichen Codierenden gewährleistet.

Für eine Übertragbarkeit und Verallgemeinerung der analysierten Ergebnisse werden 87 Interviews herangezogen und die Analysen durch eine Expert/-innengruppe begleitet.

\section{Ergebnisse}

Bei den ersten neun Samplings sind nach Abschätzung der Expert/-innengruppe (Abschn. 2.3) sieben Interviews ausreichend, um davon ausgehen zu können, dass weitere Interviews keine essentiellen Erkenntnisse mehr erbringen. Aufgrund der Heterogenität des zehnten Samplings kommt man überein, dass nach 15 Interviews die formulierten Ziele erreicht sind.

Die Ergebnisse der Interviews pro Sampling führen zu leichten Modifikationen des Interviewleitfadens, und zwar ausschließlich bezogen auf zusätzliche Unterfragen, die den Interviewten die Beantwortung erleichtern sollen.

Die Forschungsfrage wirft die Frage auf, ob ein Kategoriensystem entwickelt werden kann, das Belastungsfaktoren und Ressourcen berufsübergreifend erfasst, um damit die Daten statistischen Modellen zugänglich zu machen. Im Rahmen dieser Forschungsarbeit werden zwei Kategoriensysteme basierend auf den Quellen von Belastung und Ressourcen entwickelt, überprüft und angepasst. Nach jeder Interviewreihe erfolgt die Einordnung der Belastungsfaktoren und der Ressourcen in ein Kategoriensystem durch die unter Abschn. 2.3 beschriebene Expert/-innengruppe. Das ursprüngliche Kategoriensystem sieht die in der arbeitswissenschaftlichen Literatur oftmals verwendeten Quellen Arbeitsaufgabe, -umgebung, -organisation und -ablauf, -mittel und -platz vor. Die Kategorisierung bereits der ersten Berufsgruppe verdeutlicht, dass Belastungsfaktoren und Ressourcen die gleichen Quellen aufweisen. Dies wird durch die folgenden Interviewreihen bestätigt. Weiterhin zeigt sich, dass die Kategoriensysteme ergänzt werden müssen, um alle Aussagen der Proband/-innen kategorisieren zu können. Neben der Arbeitsaufgabe wird die Arbeitstätigkeit aufgenommen und neben dem Arbeitsplatz auch der Arbeitsraum sowie gesellschaftliche Faktoren. Dieses Kategoriensystem kann durch die letzte Interviewreihe (gemischtes Sampling) bestätigt werden. Alle getroffenen Aussagen in den Interviews können sinnvoll in die Kategoriensysteme eingeordnet werden. Jedoch zeigt sich eine Schwierigkeit, die in den Interviews genannten Faktoren 
Tab. 4 Entwickelte Kategorien für Quellen von Belastungsfaktoren und Ressourcen Table 4 Developed categories for sources of stressors and resources

\begin{tabular}{|c|c|c|}
\hline Kategorien & Erklärung & Beispiele aus den Interviews \\
\hline $\begin{array}{l}\text { Arbeitsaufgabe } \\
\text { und -tätigkeit }\end{array}$ & $\begin{array}{l}\text { Arbeitsaufgabe umfasst erforderliche Aktivitäten zur Erreichung eines } \\
\text { Arbeitsergebnisses }{ }^{\mathrm{a}} \text {. Arbeitsaufgaben umfassen alle Arbeiten, die einem } \\
\text { Beschäftigten übertragen werden }{ }^{\mathrm{d}} \text {. Hierzu gehören Inhalte, Abläufe, Pro- } \\
\text { zesse, Fähigkeiten und Fertigkeiten sowie Wissen }{ }^{\mathrm{c}} \text {. Die Arbeitstätigkeit } \\
\text { umfasst alle untergeordneten Handlungen, die der Erfüllung der Aufgabe } \\
\text { dienen und beschreibt somit die Kombination der gesamten menschlichen } \\
\text { Arbeitshandlung }\end{array}$ & $\begin{array}{l}\text { Belastungsfaktoren } \\
\text { Meine Arbeit ist körperlich anstrengend } \\
\text { Arbeitstätigkeit erfordert Daueraufmerk- } \\
\text { samkeit } \\
\text { Ressourcen } \\
\text { Interessante Aufgaben } \\
\text { Vorbereitung im Studium auf Inklusion }\end{array}$ \\
\hline Arbeitsumgebung & $\begin{array}{l}\text { Faktoren, die einen Arbeitenden umgeben }{ }^{\mathrm{a}} \text {. Zu den physikalischen Be- } \\
\text { dingungen gehören Lärm, Klima, Beleuchtung, Akustik }{ }^{\mathrm{c}} \text {, mechanische } \\
\text { Schwingungen, Strahlung, Arbeitsstoffe } \mathrm{e}^{\mathrm{e}} \text {. Zu den sozialen zählen beispiels- } \\
\text { weise Unternehmenskultur, Betriebsklima }\end{array}$ & $\begin{array}{l}\text { Belastungsfaktoren } \\
\text { Schallpegel in der Klasse } \\
\text { Mit den Kolleg/-innen muss man sich gut } \\
\text { stellen } \\
\text { Ressourcen } \\
\text { Kollegiale Unterstützung und Zusam- } \\
\text { menarbeit } \\
\text { Anerkennung durch Vorgesetzte }\end{array}$ \\
\hline $\begin{array}{l}\text { Arbeitsplatz und } \\
\text {-raum }\end{array}$ & $\begin{array}{l}\text { Arbeitsplatz beschreibt die Kombination und räumliche Anordnung der } \\
\text { Arbeitsmittel innerhalb der Arbeitsumgebung unter den durch die Arbeits- } \\
\text { aufgaben erforderlichen Bedingungen }^{\mathrm{a}} \text {, wie beispielsweise ein verstellbarer } \\
\text { Bürotisch }{ }^{\mathrm{c}} \text {. Der Arbeitsraum ist der Bereich, dem eine Person zur Durch- } \\
\text { führung der Arbeitsaufgabe zugeordnet } \text { wird }^{\mathrm{a}}\end{array}$ & $\begin{array}{l}\text { Belastungsfaktoren } \\
\text { Geringe Stellfläche } \\
\text { Fläche Arbeitsraum } \\
\text { Ressourcen } \\
\text { Ausreichend Besprechungsräume } \\
\text { Ein eigener Arbeitsplatz }\end{array}$ \\
\hline $\begin{array}{l}\text { Arbeitsmittel und } \\
\text { Schnittstellen }\end{array}$ & $\begin{array}{l}\text { Arbeitsmittel sind Werkzeuge einschließlich Hard- und Software, Maschi- } \\
\text { nen, Fahrzeuge, Geräte, Möbel, Einrichtungen ... }{ }^{a} \text { beispielsweise Headsets, } \\
\text { elektronische Schrauber, Ausstattung }{ }^{\mathrm{c}}\end{array}$ & $\begin{array}{l}\text { Belastungsfaktoren } \\
\text { Die Ausstattung ist veraltet und zu gering } \\
\text { System stürzt ab } \\
\text { Ressourcen } \\
\text { Verbesserung der materiellen Ausstattung } \\
\text { Moderne Arbeitsgeräte }\end{array}$ \\
\hline $\begin{array}{l}\text { Arbeitsorganisation } \\
\text { und -ablauf }\end{array}$ & $\begin{array}{l}\text { Arbeitsorganisation sind abgestimmte Handlungen hinsichtlich der Festle- } \\
\text { gung der Gestalt und der Arbeitsweise der Organisation, z. B. Einzel- oder } \\
\text { Teamarbeit, Ressourcenzuteilung, Mittel und Wege der Kommunikation }{ }^{\mathrm{a}} \text {, } \\
\text { Wegezeiten, Erreichbarkeit von Arbeitsmaterialien, Ablauf, Aufgaben, } \\
\text { Job Rotation, Pausensystem, Gruppenarbeit }{ }^{\mathrm{c}} \text {. Arbeitsablauf umfasst die } \\
\text { räumliche und zeitliche Abfolge des Zusammenwirkens von Arbeitenden, } \\
\text { Arbeitsmitteln, Materialien, Energie und Information a aber auch Zustän- } \\
\text { digkeiten, Informations- und Kommunikationswege, Arbeitsplanung, Ar- } \\
\text { beitszeit, Pausenregelung }\end{array}$ & $\begin{array}{l}\text { Belastungsfaktoren } \\
\text { Arbeiten am Wochenende und von zu- } \\
\text { hause } \\
\text { Es mangelt an klaren Anweisungen } \\
\text { Ressourcen } \\
\text { Flexible Arbeitszeiten (Gleitzeit/ } \\
\text { Homeoffice) } \\
\text { Personalaufstockung durch zusätzliche } \\
\text { Einstellungen }\end{array}$ \\
\hline $\begin{array}{l}\text { Gesellschaftliche } \\
\text { Faktoren }\end{array}$ & 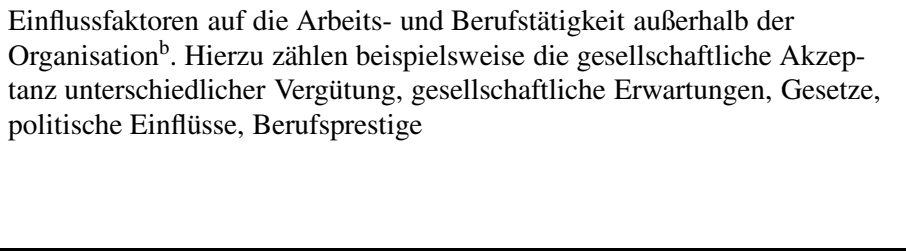 & $\begin{array}{l}\text { Belastungsfaktoren } \\
\text { Die Bezahlung ist zu niedrig } \\
\text { Zu häufiges Wechseln der Rolle } \\
\text { Ressourcen } \\
\text { Faire Bezahlung } \\
\text { Anerkennung der Leistung durch die } \\
\text { Gesellschaft }\end{array}$ \\
\hline
\end{tabular}

aDIN EN ISO 6385 (2016)

${ }^{\mathrm{b}}$ DIN EN ISO 10075-1 (2018)

cNerdinger et al. (2019)

${ }^{\mathrm{d}}$ Refa-consulting.de

${ }^{\mathrm{e} S}$ Schlick et al. (2018)

eindeutig der Arbeitsaufgabe und/oder der Arbeitstätigkeit zuzuweisen, da die Versuchspersonen selbst nicht eindeutig trennen (können), weshalb diese beiden Kategorien in einem letzten Schritt zu einer Kategorie zusammengeführt werden.

Die Kategoriensysteme sehen die in Tab. 4 erklärten und mit Beispielen aus den Interviews hinterlegten Subkategorien sowohl für Quellen von Belastungsfaktoren als auch für Quellen von Ressourcen vor.

\section{Diskussion}

Mit psychischer Belastung wird sich oftmals aufgrund zweier Fakten beschäftigt: Die Unternehmen brauchen Unterstützung bei der Erhebung psychischer Belastung und die Wissenschaft aber auch das Dienstleistungsgewerbe stellen Fragebögen zur Verfügung, um den Bedarf zu decken. Dabei wird davon ausgegangen, dass die Befragten am besten wüssten, wann eine psychische Belastung $\mathrm{zu}$ 
hoch ist und zu einer Fehlbelastung führt. Dies jedoch ist bei der Komplexität des Gesamtzusammenhangs von Interaktionen und Bedingungszusammenhängen der Belastungsfaktoren auszuschließen und somit auch einschränkend für die vorliegenden Erhebungen zu erwähnen. Diesen Umstand umschreibt auch Nachreiner (1981) mit der Vermutung, dass das Beurteilungsergebnis des Eigenzustandes hinsichtlich wahrgenommener Belastung möglicherweise mehr als die Summe der Einzelwirkungen sein könnte. Es ist von Bedeutung, dass psychische Belastung als solche erfasst und bewertet wird, nicht die subjektive Wahrnehmung oder Einschätzung der Beschäftigten. Scheiter et al. (2020) merken hierzu an, dass die subjektive Bewertung von Anstrengung und Leistung bei der Arbeit auf Heuristiken und Verzerrungen beruhen könnte und möglicherweise nicht zwangsläufig mit den zur Verfügung stehenden kognitiven Ressourcen zur Aufgabenbewältigung verknüpft sind. Selbstverständlich ist eine Fehlbelastung nur über die psychischen und physischen Reaktionen der Beschäftigten überhaupt festzustellen, sodass die Erforschung von (Fehl)Belastung nur bei Betrachtung der negativen Beanspruchungsfolgen (Fehlbeanspruchung) möglich ist. Jedoch dürfen Befragungen niemals die einzige Basis einer Belastungsstudie sein, sie können jedoch ein Beginn zu einer erweiterten Studie sein. Im Folgenden werden die Ergebnisse der bisherigen Forschungsarbeit diskutiert, Limitationen aufgezeigt sowie Implikationen ausgewiesen. Es sollte jedoch als klares Ergebnis formuliert werden, dass die Komplexität psychischer (Fehl)Belastung ausschließlich durch multidimensionale Messansätze zu betrachten ist. Hierbei jedoch muss mit der Erfassung der einzelnen, in dieser Arbeit als Belastungsfaktoren bezeichneten Aspekte begonnen werden, was Ziel dieses Teils der Studie ist.

Neben der Validierung der Kategoriensysteme zeigen die Interviews noch weitere relevante Ergebnisse. Es gibt Belastungsfaktoren, die sehr berufsspezifisch sind, wie beispielsweise „Kommunikation mit Erziehungsberechtigten“ oder „Forschungsbericht verfassen“. Andere Belastungsfaktoren sind berufsübergreifend. Hierbei sind besonders Belastungsfaktoren auffällig, die zeitliche Vorgaben umfassen. Diese kamen in allen geführten Interviews vor. Es zeigt sich in den Interviews ebenso, dass die Versuchspersonen unterschiedliche Auffassungen und Definitionen von beispielsweise Zeit- bzw. Termindruck haben. Dennoch können die Interviewergebnisse in drei Kategorien unterteilt werden:

Zeitdruck beispielsweise „Stoßzeiten“ (Gastgewerbe) oder „Zeitdruck“ (Wissenschaftliche Mitarbeitende)

Parallelaufgaben beispielsweise „Viele Menschen wollen gleichzeitig etwas von mir“ (Gastgewerbe), „Mehrere Anforderungen treten gleichzeitig auf“ (IT) oder „Dinge passieren gleichzeitig" (Pflegepersonal), sowie
Informationsmenge beispielsweise „Hoher Informationsaustausch“ (Lehrende 1) oder „Immer aufmerksam sein“ (Lehrende 2).

Ob Nennungen wie „Arbeitsmenge“ oder „Mehrarbeit“ unter Zeitdruck, Parallelaufgaben und Informationsmenge fallen, muss in weiteren Befragungen geklärt werden. Hierzu bedarf es jedoch einer genauen Definition dessen, was erhoben werden soll. Umgangssprachlich ist "Zeitdruck“ für jeden verständlich, Tatsache ist jedoch, dass Zeitdruck eine Belastungsfolge ist, keine Belastung. Die Belastung wäre beispielsweise die Zeitvorgabe für eine Tätigkeit. Für die weiterführende Forschungsarbeit werden die folgenden Definitionen entwickelt:

Zeitdruck: Diskrepanz von zur Verfügung stehender Zeit und der erforderlichen Zeit zur Bewältigung von Aufgabe(n) in angemessener und regulärer Arbeitsgeschwindigkeit.

Parallelaufgaben: Abarbeitung wechselnder, sich zeitlich überlappender, diskreter Aufgaben und Tätigkeiten innerhalb eines vorgegebenen Zeitrahmens.

Informationsmenge: Diskrepanz zwischen eingehender Information und Kapazitäten der Informationsaufnahme und -verarbeitung innerhalb eines vorgegebenen Zeitrahmens.

\subsection{Fazit}

Die grundlegende Frage der vorliegenden Forschungsarbeit ist, ob Grenzwerte psychischer Belastung eindeutig festgelegt werden können. Da diese Frage, wie die vorliegenden Ausführungen aufgezeigt haben, hochgradig komplex ist, muss in einem ersten Schritt ein Arbeitsmodell zur Untersuchung der künftigen Fragestellungen erstellt und die Komponenten definiert werden sowie Kategoriensysteme entwickelt werden, die die Erhebung der Belastungsfaktoren sowie der Ressourcen und deren Umsetzung in statistische Modelle ermöglicht.

Die Forschungsfrage der vorliegenden Untersuchung befasst sich damit, ob es ein berufsgruppenübergreifendes Kategoriensystem gibt, um Belastungsfaktoren und Ressourcen zu erheben. Diese Frage ist relevant, um vorhandene und künftige Daten einem statistischen Vorhersagemodell zuführen zu können und somit die Forschungsfrage „Wann wird psychische Belastung zu Fehlbelastung“ untersuchen zu können. Ebenfalls kann so die Frage beantwortet werden, ob und wie genau Ressourcen Belastung optimieren können.

Die Interviews haben zweierlei gezeigt. Einerseits sind die Quellen von Belastungsfaktoren ebenfalls die Quellen der möglichen Ressourcen. Dies ist ein wichtiger Punkt bei der weiteren Betrachtung der erhobenen Daten, denn 
es erfordert eine deutliche definitorische Abgrenzung der Aspekte, die erhoben werden. Hierbei sollte die angesprochene Problematik untersucht werden, ob Ressourcen Belastungsfaktoren sind oder diese lediglich beeinflussen.

Andererseits wird die Entwicklung von Kategoriensystemen dargestellt, in die alle bisher erhobenen Daten sinnvoll eingeordnet werden können. Die Forschungsfrage kann nach dem heutigen Stand des Wissens demzufolge bejaht werden. Allerdings ist abzuwarten, ob es nicht noch weitere Berufsgruppen gibt, die Belastungsfaktoren und Ressourcen nennen, die nicht in diese Kategoriensysteme eingeordnet werden können. In diesem Fall müssten die Kategoriensysteme überarbeitet werden. Das Kategoriensystem der Belastungsfaktoren ist sehr eng an die Definition psychischer Belastung angelehnt. Es fällt daher in den Untersuchungen auf, dass solche „Belastungsfaktoren“, die nicht einzuordnen sind, tatsächlich Beanspruchungen sind. Eine Unterscheidung - die der Laie nicht treffen kann - ist hier besonders relevant.

Die Kategoriensysteme sind wissenschaftlich gut fundiert und in der Empirie sinnvoll einzusetzen. In der empirischen Arbeit hat sich jedoch herauskristallisiert, dass die Kategorien und deren Subkategorien noch prägnanter definiert werden müssen, da unterschiedliche Beurteilende teilweise zu unterschiedlichen Urteilen kommen. Das kann nicht das Ziel einer Kategorisierung sein. Eine prägnantere Definition mit weniger individuellen Freiheitsgraden bei der Beurteilung muss demzufolge noch vorgenommen werden.

\subsection{Limitationen der Datenerhebung}

Die Gütekriterien qualitativer Forschung werden sowohl bei der Datenerhebung als auch bei der Datenauswertung berücksichtigt. Dennoch sind Limitationen zu nennen.

Die Interviewleitfragen beinhalten Suggestivanteile, wie beispielsweise die Wortwahl „nervig“. Dies kann kritisiert werden, jedoch ist davon auszugehen, dass Versuchspersonen, die sich nicht mit dem Belastungs-BeanspruchungsKonzept auseinandersetzen, keine Unterscheidung zwischen Belastungsfaktoren und Fehlbelastung treffen können, weshalb diese Begriffe in Alltagssprache präsentiert werden. Die letzte Folgefrage löst jedoch diese scheinbare Diskrepanz auf und klärt die Begrifflichkeiten, um nochmals einen anderen Blickwinkel auf den abzufragenden Sachverhalt zu erlauben.

Ebenfalls kann die Anzahl der zu führenden Interviews kritisiert werden. Die Setzung von mindestens sieben Interviews erfolgt aufgrund Interviewerfahrungen aus der Expert/-innengruppe. Diese Gruppe entscheidet darüber, ob weitere Interviews zu führen sind. Dennoch kann nicht ausgeschlossen werden, dass weitere Interviews zu mehr branchenspezifischen Belastungsfaktoren führen.

\subsection{Ausblick}

Es muss ausdrücklich darauf hingewiesen werden, dass dieser Teil der Studie erst der „Aufschlag“ zu einer Reihe von Studien ist, um zu klären, ob und wie die Bestimmung von Grenzwerten psychischer Belastung möglich ist. Wenn Grenzwerte psychischer Belastung definiert werden können, dann muss dies für genau zu definierende prospektive Zielpopulationen gelten.

Die Forschungsarbeit wird derzeit durch fortlaufende Untersuchungen ergänzt, beispielsweise indem durch weitere Berufsgruppen die Kategoriensysteme einer wiederholten empirischen Überprüfung unterzogen werden und indem die Subkategorien der Kategoriensysteme detaillierter definiert werden, um Auswertungsobjektivität zu schaffen.

Weiterhin werden Daten zu psychischer Belastung und Fehlbelastung durch physiologische/neuronale Messungen, Fehlleistungen, Befragungen und Beobachtung sowohl im experimentellen Rahmen (aktuell mit dem Stroop-Test unter Zeitdruckbedingungen sowie mit einer digitalen Postkorbübung) sowie in Felduntersuchungen (beispielsweise Arbeitsabläufe) erhoben. Hilfreiche Unterstützung bietet eine weitere Richtung der Forschungsarbeit, die sich mit Betrachtung von Verhaltensweisen (im speziellen Mimik) und Fehlerraten als Ausdruck von psychischer Belastung auseinandersetzt (Ferreira et al. 2022).

Abschließend muss auf die Notwendigkeit hingewiesen werden, den Engpass der kognitiven und emotionalen Informationsverarbeitungskapazität in den Fokus künftiger Untersuchungen zu nehmen. Wie die Interviews zeigen, werden die in der Diskussion definierten Belastungsfaktoren Zeitdruck, Parallelaufgaben und Informationsmenge von allen Interviewten angeführt. Auch müssen die bereits erwähnten weitreichenden Änderungen in den Arbeitsbedingungen bei den Betrachtungen mit einbezogen werden, wie beispielsweise die technische Automatisierung von Arbeitsprozessen, die zu einer Änderung der notwendigen menschlichen Informationsverarbeitung führt und damit ebenfalls Auswirkungen auf die Aufgabenkomplexität und mögliche Engpässe hat (Jipp und Steil 2021). Dieser Fokus auf den Engpass der kognitiven und emotionalen Informationsverarbeitungskapazität ist bedeutsam und unerlässlich für die Betrachtung von Grenzwerten psychischer Belastung.

Mit einem letzten Blick auf die Frage, wie wissenschaftlich fundierte Grenzwerte psychischer Belastung aussehen könnten, möchte die Autorenschaft gerne eine Vision teilen. In der Differentiellen Psychologie (aber auch in anderen Bereichen der psychologischen Diagnostik) existieren valide Erhebungsinventare für beispielsweise Leistungs- und Persönlichkeitstests. Basis all dieser Tests ist eine angemessene Vergleichspopulation und die Übertragung der Testwerte 
in Normskalen (Stanine, Prozentwerte, z-Werte, SW-Werte ...). Die empirisch erhobenen Daten werden somit aufbereitet, um über durchschnittliche, unter- oder überdurchschnittliche Werte Auskunft geben zu können. Wenn es möglich wäre, von Fehlbeanspruchung auf Schädigung zu schließen und basierend darauf auf Gefährdung und Fehlbelastung können für einzelne Belastungsfaktoren über die Vergleichspopulation Normbereiche angegeben werden, die (wahrscheinlich) nicht zu aversiven Folgen im Sinne einer Fehlbeanspruchung führen.

Bei diesem Vorgehen sind mindestens drei Einschränkungen zu nennen:

1. Über das Abbilden von (empirisch ermittelten) Normbereichen können Belastungsfaktoren für - je nach Zielsetzung - einen Großteil einer vorab bestimmten menschlichen Zielpopulation festgelegt werden, nicht aber für alle Personen

2. Die Problematik der Superpositionen ist mit dieser Vorgehensweise (noch) nicht berücksichtigt

3. Empirisch ermittelte Normbereiche werden letztendlich politisch diskutiert und verändert, um sie umsetzbar zu machen.

Dennoch würde wenigstens erstmals eine wissenschaftliche fundierte Datenbasis als Beitrag zu dieser Diskussion vorhanden sein.

Danksagung Die Autorenschaft bedankt sich herzlich bei Prof. Dr. Friedhelm Nachreiner für die zielführenden und konstruktiven Diskussionen.

Funding Open Access funding enabled and organized by Projekt DEAL.

Open Access Dieser Artikel wird unter der Creative Commons Namensnennung 4.0 International Lizenz veröffentlicht, welche die Nutzung, Vervielfältigung, Bearbeitung, Verbreitung und Wiedergabe in jeglichem Medium und Format erlaubt, sofern Sie den/die ursprünglichen Autor(en) und die Quelle ordnungsgemäß nennen, einen Link zur Creative Commons Lizenz beifügen und angeben, ob Änderungen vorgenommen wurden.

Die in diesem Artikel enthaltenen Bilder und sonstiges Drittmaterial unterliegen ebenfalls der genannten Creative Commons Lizenz, sofern sich aus der Abbildungslegende nichts anderes ergibt. Sofern das betreffende Material nicht unter der genannten Creative Commons Lizenz steht und die betreffende Handlung nicht nach gesetzlichen Vorschriften erlaubt ist, ist für die oben aufgeführten Weiterverwendungen des Materials die Einwilligung des jeweiligen Rechteinhabers einzuholen.

Weitere Details zur Lizenz entnehmen Sie bitte der Lizenzinformation auf http://creativecommons.org/licenses/by/4.0/deed.de.

\section{Literatur}

ArbSchG Arbeitsschutzgesetz (2015) Gesetz über die Durchführung von Maßnahmen des Arbeitsschutzes zur Verbesserung der Sicherheit und des Gesundheitsschutzes der Beschäftigten bei der Arbeit, S. 1-11. Stand 8. Sept. 2015. https://www.bmas.de/
DE/Service/Gesetze-und-Gesetzesvorhaben/arbeitsschutzgesetz. html. Zugegriffen: 25. Nov. 2021

Asendorpf J (2009) Personality: traits and situations. In: Corr P, Matthews $\mathrm{G}$ (Hrsg) The cambridge handbook of personality psychology. Cambridge University Press, Cambridge, S 43-53

Bakker AB, Demerouti E (2007) The job demands-resources model: state-of-the-art. Journal of Managerial Psych 22(3):309-328

Bamberg E, Mohr G (2016) Psychologisches Wissen für die Praxis: Gefährdungsbeurteilungen im Arbeits- und Gesundheitsschutz. Psychol Rundsch 67:130-134. https://doi.org/10.1026/ 0033-3042/a000314

Bauer W, Mütze-Niewöhner S, Stowasser S, Zanker C, Müller N (2021) Arbeit in der digitalisierten Welt: Praxisbeispiele und Gestaltungslösungen aus dem BMBF-Förderschwerpunkt. Springer Nature, Berlin

Beck D, Richter G, Ertel M, Morschhäuser M (2012) Gefährdungsbeurteilung bei psychischen Belastungen in Deutschland - Verbreitung, hemmende und fördernde Bedingungen. Praev Gesundheitsf 2:115-119

Böcker M, Vogt J, Christ O, Müller-Leonhardt A (2009) Health, safety, and performance in high altitude observatories: a sustainable approach. Messenger 137:33-35

Bornewasser M, Wegge J (2018). Editorial Moderne Stressdiagnostik am Arbeitsplatztheoretische und methodische Fortschritte. Wirtschaftspsychologie (1):3-11

Bouveresse L, Chanlat J-F, Baujard C (2011) Occupational stress on French customer service employees: impact of human ressource management modes. Research Advances in Organizational Behavior and Human Resources Management CNRS and Paris Dauphine DRM University, S 164-181

Bundesrat Drucksache 315/13 (2014) Verordnung zum Schutz vor Gefährdungen durch psychische Belastung bei der Arbeit. https:// www.bundesrat.de/SharedDocs/beratungsvorgaenge/2013/03010400/0315-13.html. Zugegriffen: 21. Aug. 2020

Cernavin O, Schröter W, Stowasser S (2018) Prävention 4.0. Analysen und Handlungsempfehlungen für eine produktive und gesunde Arbeit 4.0. Springer Nature, Wiesbaden

Deutsche Gesetzliche Unfallversicherung (2013) Gefährdungsbeurteilung psychischer Belastungen. Tipps zum Einstieg. IAG Report $1 / 2013$

DIN EN ISO 10075-1 (2018) Ergonomische Grundlagen bezüglich psychischer Arbeitsbelastung - Teil 1: Allgemeine Aspekte und Konzepte und Begriffe (ISO 10075-1:2017); Deutsche Fassung EN ISO 10075-1:2017. Beuth, Berlin

DIN EN ISO 6385 (2016) Grundsätze der Ergonomie für die Gestaltung von Arbeitssystemen. Deutsche Fassung DIN EN ISO 6385:2016. Ersatz für DIN EN ISO 6385: 2004, 5. Beuth, Berlin

Ferreira Y, Ata E, Müzeyyen A, Vogt J (2022) Messung psychischer Belastung über Verhaltensweisen und Fehlerraten. 68. GfA-Frühjahrskongress, 2022. Technologie und Bildung in hybriden Arbeitswelten, Magdeburg, 02.03.-04.03.2022 (eingereicht und angenommen)

GDA - Gemeinsame Deutsche Arbeitsschutzstrategie (2018) Arbeitsschutz gemeinsam anpacken. Leitlinie Beratung und Überwachung bei psychischer Belastung am Arbeitsplatz. Herausgeber: Geschäftsstelle der Nationalen Arbeitsschutzkonferenz, Berlin. http://www.gda-portal.de/DE/Downloads/pdf/Leitlinie-PsychBelastung.pdf?_blob=publicationFile. Zugegriffen: 15. Juni 2020

Haas R, Reblin S (2021) Bio-psycho-soziales betriebliches Gesundheitsmanagement für Sozial- und Gesundheitsberufe. Ernst Reinhardt, München

Hacker W (1984) Psychologische Bewertung von Arbeitsgestaltungsmaßnahmen. Springer, Berlin, Heidelberg

Hacker W (1986) Arbeitspsychologie: Psychische Regulation von Arbeitstätigkeiten. VEB Deutscher Verlag der Wissenschaften, Berlin 
Hobfoll SE, Buchwald P (2004) Die Theorie der Ressourcenerhaltung und das multiaxiale Copingmodell - eine innovative Stresstheorie. In: Buchwald $\mathrm{P}$, et al. (Hrsg) Stress gemeinsam bewältigen. Ressourcenmanagement und multiaxiales Coping. Hogrefe, Göttingen, S 11-26

ISO 10075 (1991) Ergonomic principles related to mental workload-Part 1: General terms and definitions. www.iso.org

Jipp M, Steil J (2021) Steuern wir oder werden wir gesteuert? Chancen und Risiken von Mensch-Technik-Interaktion. In: Haux R, Gahl K, Jipp M, Kruse R, Richter O (Hrsg) Zusammenwirken von natürlicher und künstlicher Intelligenz. Springer VS, Wiesbaden, S 17-34

Joiko K, Schmauder M, Wolff G (2010) Psychische Belastung und Beanspruchung im Berufsleben. Erkennen - Gestalten. Hrgs. BAuA - Bundesanstalt für Arbeitsschutz und Arbeitsmedizin. https:// www.baua.de/DE/Angebote/Publikationen/Praxis/A45.pdf? blob=publicationFile. Zugegriffen: 15. Juni 2020

Junghanns G, Morschhäuser M, Bundesanstalt für Arbeitsschutz und Arbeitsmedizin (2013) Immer schneller, immer mehr: psychische Belastung bei Wissens- und Dienstleistungsarbeit. Springer VS, Wiesbaden

Karasek R (1979) Job demands, job decision latitude, and mental strain: Implications for job redesign. Adm Sci Q. https://doi.org/ $10.2307 / 2392498$

Krehl C, Balfe N (2014) Cognitive workload analysis in rail signalling environments. Cognition, Technology \& Work 16(3):359-371. https://doi.org/10.1007/s10111-013-0266-7

Kuckartz U (2018) Qualitative Inhaltsanalyse. Methoden, Praxis, Computerunterstützung. Beltz Juventa, Weinheim, Basel

LASI (2002) Konzept zur Ermittlung psychischer Fehlbelastungen am Arbeitsplatz und zu Möglichkeiten der Prävention. LV 28. https:// lasi-info.com/uploads/media/lv28_01.pdf. Zugegriffen: 24. Juli 2020

Lazarus RS, Folkman S (1984) Stress, appraisal, and coping. Springer, New York

Luczak H, Rohmert W (1997) Belastungs-Beanspruchungskonzepte. In: Luczak H, Volpert W (Hrsg) Handbuch Arbeitswissenschaft. Schäffer-Poeschel, Stuttgart, S 326-332

McGrath JE (1981) Stress und Verhalten in Organisationen. In: Nitsch JR (Hrsg) Stress. Huber, Bern, S 441-499

Möller K, Gamm N, Braun M, Iserloh B, Kastner M, Kliesch G, Köper B, Pennig S, Vogt J (2008) Strategische Steuerung der betrieblichen Gesundheitsförderung mit Strategy Maps. Z Manag 3:247-280

Nachreiner F (1981) Eigenzustandsskalierung zur Integration von Beanspruchungen. Vortragsmanuskript zur Herbstkonferenz der GfA, 2. Okt. 1981

Nachreiner F (2008) Erfassung psychischer Belastung und Rückwirkung auf die Arbeitsgestaltung. Leistung und Lohn: Zeitschrift für Arbeitswirtschaft 445/449:7-28

Nerdinger FW, Blickle G, Schaper N (2019) Arbeits- und Organisationspsychologie. Springer, Heidelberg

Oesterreich R (2001) Das Belastungs-Beanspruchungskonzept im Vergleich mit arbeitspsychologischen Konzepten. Z Arb Wiss 55(3): 162-178

Paridon H, Mühlbach J (2016) Psychische Belastung in der Arbeitswelt - Eine Literaturanalyse zu Zusammenhängen mit Gesundheit und Leistung. IGA Report, Bd. 32. AOK-Bundesverband, BKK Dachverband e. V., Deutsche Gesetzliche Unfallversicherung (DGUV), Verband der Ersatzkassen e. V. (vdek), Initiative Gesundheit und Arbeit

Pennig S, Vogt J (2008) Profitability assessment and economic evaluation of human performance management programmes-guidelines and tools. Bundesanstalt für Arbeitsschutz und Arbeitsmedizin, Dortmund
REFA-Institut (2016) Arbeitsorganisation erfolgreicher Unternehmen - Wandel in der Arbeitswelt. Hanser

Richardi R (Hrsg) (2017) Arbeitsgesetze. Mit den wichtigsten Bestimmungen zum Arbeitsverhältnis, Kündigungsrecht, Arbeitsschutzrecht, Berufsbildungsrecht, Tarifrecht, Betriebsverfassungsrecht, Mitbestimmungsrecht und Verfahrensrecht: Textausgabe mit ausführlichem Sachverzeichnis (dtv Beck-Texte im dtv, Bd. 5006, 91., neu bearbeitete Auflage, Stand: 1. Juli 2017, Sonderausgabe). dtv, München

Richter G, Kuhn K (2010) Toolbox Version 1.2. Instrumente zur Erfassung psychischer Belastungen. Projekt F 1965. Bundesanstalt für Arbeitsschutz und Arbeitsmedizin, Dortmund, Berlin, Dresden

Richter P, Hacker W (1998) Belastung und Beanspruchung: Stress, Ermüdung und Burnout im Arbeitsleben. Asanger, Heidelberg

Rohmert W (1972) Aufgaben und Inhalt der Arbeitswissenschaft. Die Berufsbildende Sch 24/1972:3-14

Rohmert W (1984) Das Belastungs-Beanspruchungs-Konzept. Z Arb Wiss 38(10 NF):193-200

Rohmert W, Rutenfranz J (1975) Arbeitswissenschaftliche Beurteilung der Belastung und Beanspruchung an unterschiedlichen industriellen Arbeitsplätzen. Der Bundesminister für Arbeit und Sozialordnung, Bonn

Rothe I, Adolph L, Beermann B, Schütte M, Windel A, Grewer A, Lenhardt U, Michel J, Thomson B, Formazin M (2017) Psychische Gesundheit in der Arbeitswelt. Wissenschaftliche Standortbestimmung. Forschung Projekt F2353.Bundesanstalt für Arbeitsschutz und Arbeitsmedizin. https://www.baua.de/DE/ Angebote/Publikationen/Berichte/Psychische-Gesundheit.pdf? blob=publicationFile. Zugegriffen: 12. Okt. 2021

Scheiter K, Ackerman R, Hoogerheide V (2020) Looking at mental effort appraisals through a metacognitive lens: are they biased? Educ Psychol Rev. https://doi.org/10.1007/s10648-020-09555-9

Schlick C, Bruder R, Luczak H (2018) Arbeitswissenschaft. Springer, Berlin, Heidelberg

Schmidt K-H, Diestel S (2013) Jobs demands and personal resources in their relations to indicators of job strain among nurses for older people. J Adv Nurs. https://doi.org/10.1111/jan.12082

Schubert FC, Knecht A (2015) Ressourcen - Merkmale, Theorien und Konzeptionen im Überblick: eine Übersicht über Ressourcenansätze in Soziologie, Psychologie und Sozialpolitik. http://www. ssoar.info/ssoar/handle/document/50698. Zugegriffen: 11. Feb. 2020

Schuller K (2020) Interventions as the centrepiece of psychosocial risk assessment-why so difficult? IJWHM 13(1):61-80

Uhle T, Treier M (2015) Betriebliches Gesundheitsmanagement: Gesundheitsförderung in der Arbeitswelt - Mitarbeiter einbinden, Prozesse gestalten, Erfolge messen. Springer, Heidelberg

Ulich E (2005) Arbeitspsychologie, 6. Aufl. Schäffer-Poeschel, Stuttgart

Vogt J, Adolph L, Ayan T, Udovic A, Kastner M (2002) Stress in modern air traffic control systems and potential influences on memory performance. J Hum Factors Aerosp Saf 2(4):355-378

Vogt J, Hagemann T, Kastner M (2006) The impact of workload on heart rate and blood pressure in en-route and tower air traffic control. J Psychophysiol 20(4):297-314. https://doi.org/10.1027/ 0269-8803.20.4.297

Vogt J, Leonhardt J, Köper B, Pennig S (2010) Human factors in safety and business management. Ergonomics 53(2):149-163

Wickens CD (2008) Multiple resources and mental workload. Hum Factors. https://doi.org/10.1518/001872008X288394

Wulf I, Süß S, Diebig M (2017) Akteure der Gefährdungsbeurteilung psychischer Belastung - Perspektiven und Konflikte im betrieblichen Arbeits- und Gesundheitsschutz. Z Arb Wiss 71:296-304 